Article

\title{
$A b$ Initio and Monte Carlo Approaches For the Magnetocaloric Effect in Co- and In-Doped Ni-Mn-Ga Heusler Alloys
}

\author{
Vladimir Sokolovskiy ${ }^{1,2}$, Anna Grünebohm ${ }^{3}$, Vasiliy Buchelnikov ${ }^{1}$ and Peter Entel ${ }^{3, *}$ \\ ${ }^{1}$ Chelyabinsk State University, Br. Kashirinykh 129, 454001 Chelyabinsk, Russia; \\ E-Mails: vsokolovsky84@mail.ru(V.S.); buche@csu.ru (V.B.) \\ ${ }^{2}$ National University of Science and Technology "MISiS", Leninskiy pr. 4, 119049 Moscow, Russia \\ ${ }^{3}$ University of Duisburg-Essen, Lotharstrasse 1, D-47048 Duisburg, Germany; \\ E-Mail: anna@thp.uni-due.de
}

* Author to whom correspondence should be addressed; E-Mail: entel@thp.uni-due.de;

Tel.: +49-203-379-3330; Fax: +49-203-379-3665.

Received: 25 August 2014; in revised form: 12 September 2014 / Accepted: 12 September 2014 /

Published: 19 September 2014

\begin{abstract}
The complex magnetic and structural properties of Co-doped Ni-Mn-Ga Heusler alloys have been investigated by using a combination of first-principles calculations and classical Monte Carlo simulations. We have restricted the investigations to systems with 0,5 and 9 at\% Co. Ab initio calculations show the presence of the ferrimagnetic order of austenite and martensite depending on the composition, where the excess $\mathrm{Mn}$ atoms on $\mathrm{Ga}$ sites show reversed spin configurations. Stable ferrimagnetic martensite is found for systems with 0 (5) at\% Co and a $c / a$ ratio of 1.31 (1.28), respectively, leading to a strong competition of ferro- and antiferro-magnetic exchange interactions between nearest neighbor Mn atoms. The Monte Carlo simulations with ab initio exchange coupling constants as input parameters allow one to discuss the behavior at finite temperatures and to determine magnetic transition temperatures. The Curie temperature of austenite is found to increase with Co, while the Curie temperature of martensite decreases with increasing Co content. This behavior can be attributed to the stronger Co-Mn, Mn-Mn and Mn-Ni exchange coupling constants in austenite compared to the corresponding ones in martensite. The crossover from a direct to inverse magnetocaloric effect in Ni-Mn-Ga due to the substitution of $\mathrm{Ni}$ by Co leads to the appearance of a "paramagnetic gap" in the martensitic phase. Doping with In increases the magnetic jump at the martensitic transition temperature. The simulated magnetic and magnetocaloric properties of Co- and In-doped Ni-Mn-Ga alloys are in good qualitative agreement with the available experimental data.
\end{abstract}


Keywords: Heusler alloys; magnetocaloric effect; first-principles and Monte Carlo methods

PACS classifications: $75.30 . \mathrm{Sg} ;$ 81.30.Kf; 75.10.-b; 31.15.A-

\section{Introduction}

Among ferromagnetic (FM) materials having acceptable magnetocaloric properties, Ni-Mn-Ga Heusler alloys have been widely investigated by experimental and theoretical methods in view of their potential applications as intelligent functional materials [1-5]. The multifunctional properties usually appear across a martensitic transformation due to the strong coupling of crystal structure and magnetic order. The shape memory effect and magnetic-field-induced strains [6-10,12], superelasticity [13-15], magnetocaloric effect [16-19], as well as the spin glass and strain glass behavior [20-23] are found among these multifunctional properties. Generally, the tuning of both, structural and magnetic transition temperatures, can be useful to achieve better functional properties. At present, this optimization problem of Heusler alloys is being intensively investigated.

There is an extreme sensitivity of the crystal structure of the martensitic phase with respect to composition in off-stoichiometric $\mathrm{Ni}_{2+x} \mathrm{Mn}_{1-x} \mathrm{Ga}$ or $\mathrm{Ni}_{2} \mathrm{Mn}_{1+x} \mathrm{Ga}_{1-x}$ alloys [21,24-28], showing that in dependence of composition and thermo-mechanical treatments, the crystal structure of low-temperature martensite can be tetragonal or monoclinic, with five-layered modulation (5M) [29], orthorhombic or monoclinic, with seven-layered modulation (7M) [30,31], or non-modulated tetragonal (NM) [32,33].

Recently, the crossover between tetragonal and orthorhombic structures in Ni-Mn-Ga with a structural transition temperature close to room temperature has been reported by Richard et al. [28]. The authors have observed that the mixed region, where both tetragonal and orthorhombic structures can coexist, is near compositions with 50 at\% $\mathrm{Ni}$ and Mn content between 28 and 30 at\%; whereas the samples with high Mn content display the orthorhombic structure (7M), while compositions closer to 50 at\% Ni show the tetragonal (5M) phase; see Figure 1. In addition, alloys like $\mathrm{Ni}_{50} \mathrm{Mn}_{30} \mathrm{Ga}_{20}\left(\mathrm{Ni}_{2} \mathrm{Mn}_{1.2} \mathrm{Ga}_{0.8}\right)$ exhibit a large magneto-crystalline anisotropy, which makes the alloy suitable for shape memory applications at room temperature, due to the exhibition of favorable structural ordering and twin-boundary motion.

A way to significantly improve the magnetic and mechanical properties of Ni-Mn-Ga alloys is related to adding a fourth element with the partial substitution of $\mathrm{Ga}$, Ni or $\mathrm{Mn}$. It should be noted that the fourth element should have a covalent radius and a valence electron concentration close to the parent elements of the alloy in order to avoid too large volume or strain effects. An interesting doping element is a transition metal element, like Co. In general, if the doping element decreases the overall valence electron concentration $e / a$, we expect that $T_{m}$ will decrease, too. Therefore, when Co $\left(3 \mathrm{~d}^{7} 4 \mathrm{~s}^{2}\right)$ substitutes for Ni $\left(3 \mathrm{~d}^{8} 4 \mathrm{~s}^{2}\right), e / a$ decreases, but is increased in the case of Co replacing $\mathrm{Mn}\left(3 \mathrm{~d}^{5} 4 \mathrm{~s}^{2}\right)$ or Ga $\left(4 \mathrm{~s}^{2} 4 \mathrm{p}^{1}\right)$. If using $\mathrm{Co}$, it is expected that the Curie temperature will increase because of the strong ferromagnetic nature of Co.

During the past decade, a series of investigations of the magnetic and structural properties of Co-doped Ni-rich and Mn-rich Ni-Mn-Ga Heusler alloys has been reported by different groups regarding the substitution of $\mathrm{Co}$ for $\mathrm{Ni}, \mathrm{Mn}$, as well as Ga. Since, in this paper, we present the results of $a b$ 
initio calculations and Monte Carlo simulations of substitutional processes (mainly $\mathrm{Co}$ for $\mathrm{Ni}$ ), we have gathered in Table 1 the investigations from the literature (without claiming to have a complete list of all investigations performed so far) [19,34-50].

Figure 1. Phase diagram of Mn-rich Ni-Mn-Ga alloys showing the region where tetragonal and orthorhombic structures may coexist at room temperatures (denoted as "mixed" [28]). The dashed line indicates compositions with $50 \% \mathrm{Ni}$. The middle eigenvalue of the transformation matrix is marked in the panel and has been derived from measurements of the lattice constant of austenite and martensite [28]. An eigenvalue close to one indicates a possible coherent lattice invariant martensite-austenite interface.

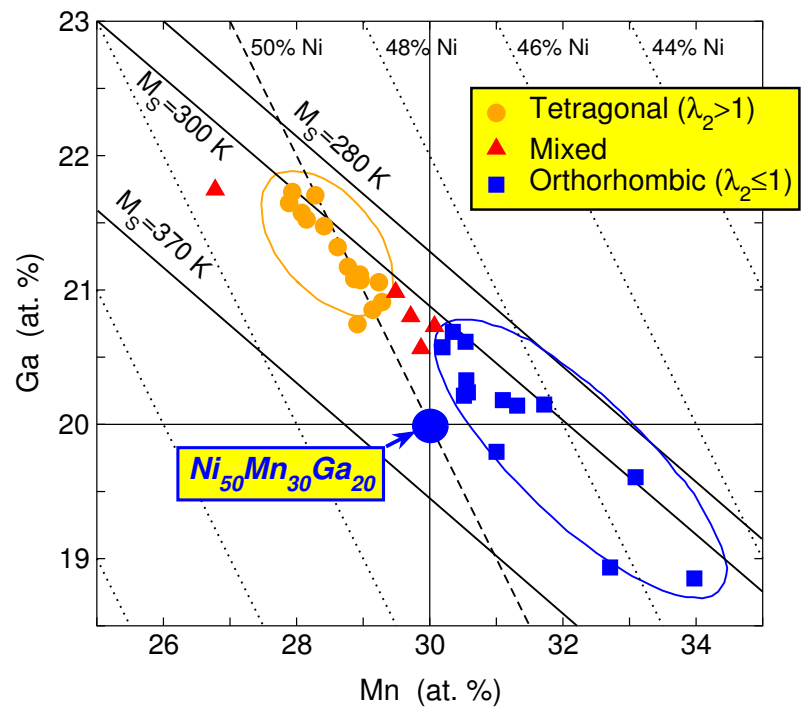

Table 1. The variety of Co-doped Ni-Mn-Ga Heusler alloys that have been recently investigated.

\begin{tabular}{lll}
\hline Substitution of Co for Ni & Substitution of Co for Mn & Substitution of Co for Ga \\
\hline $\mathrm{Ni}_{2+x-y} \mathrm{Co}_{y} \mathrm{Mn}_{1-x} \mathrm{Ga}$ & $\mathrm{Ni}_{2} \mathrm{Mn}_{1+x-y} \mathrm{Co}_{y} \mathrm{Ga}_{1-x}$ & $\mathrm{Ni}_{2} \mathrm{Mn}_{1+x} \mathrm{Ga}_{1-x-y} \mathrm{Co}_{y}$ \\
$x=0.16,0 \leq y \leq 0.09[34]$ & $x=0.16,0.0 \leq y \leq 0.26[40]$ & $x=0,0 \leq y \leq 0.25[38]$ \\
$\mathrm{Ni}_{2+x-y} \mathrm{Co}_{y} \mathrm{MnGa}_{1-x}$ & $\mathrm{Ni}_{2+x} \mathrm{Mn}_{1-y} \mathrm{Co}_{y} \mathrm{Ga}_{1-x}$ & $x=0,0 \leq y \leq 0.2[46]$ \\
$x=0.12,0 \leq y \leq 0.56[35]$ & $x=0.24,0.16 \leq y \leq 0.32[36]$ & $x=0.16, y=0.088[37]$ \\
$x=0.24,0 \leq y \leq 0.32[36]$ & $\mathrm{Ni}_{2+x} \mathrm{Mn}_{1-x-y} \mathrm{Co}_{y} \mathrm{Ga}$ & $x=0.16,0 \leq y \leq 0.17[47]$ \\
$\mathrm{Ni}_{2-y} \mathrm{Co}_{y} \mathrm{Mn}_{1+x} \mathrm{Ga}_{1-x}$ & $x=0,0 \leq y \leq 0.25[45]$ & $\boldsymbol{T}_{m}$ increases, \\
$x=0,0 \leq y \leq 0.2[38]$ & $x=0,0.03 \leq y \leq 0.2[38]$ & $\boldsymbol{T}_{C}$ weakly changes \\
$x=0.16,0 \leq y \leq 0.16[40]$ & $x=0.16,0.04 \leq y \leq 0.14[34]$ & \\
$x=0.16,0 \leq y \leq 0.13[42]$ & $\boldsymbol{T}_{m}$ increases, & \\
$x=0.16,0 \leq y \leq 0.4[44]$ & $\boldsymbol{T}_{C}$ weakly changes & \\
$x=0.24,0.20 \leq y \leq 0.24[39]$ & & \\
$0 \leq x \leq 0.11,0.068 \leq x \leq 0.232[37]$ & & \\
$0.2 \leq x \leq 0.28,0 \leq y \leq 0.36[19,41]$ & & \\
$0.2 \leq x \leq 0.28,012 \leq y \leq 0.32[43]$ & & \\
$\boldsymbol{T}_{m}$ decreases, $\boldsymbol{T}_{C}$ increases & & \\
\hline
\end{tabular}


In general, it has been established that in Ni-Mn-Ga-Co compounds, the martensitic transformation temperature is found to increase with the substitution of $\mathrm{Mn}$ or Ga by $\mathrm{Co}$ and to decrease when replacing $\mathrm{Ni}$ by Co. Hence, the variation of the martensitic transition temperature follows the general trend that $T_{m}$ is proportional to the average valence electron number per atom $(e / a)$ ratio. Concerning the Curie temperature, $T_{C}$ increases with the substitution of $\mathrm{Co}$ for $\mathrm{Ni}$ and shows a weak effect with non-monotonous behavior when $\mathrm{Mn}$ or $\mathrm{Ga}$ is replaced by Co. Interestingly, in samples with the substitution of Ga by Co, $T_{C}$ is almost independent of the doping concentration when the FM transition takes place in austenite, while it decreases with increasing $x$ when the FM order occurs within the martensite [38,46]. With respect to the critical value of Co content in the Ni sublattice for which the martensitic phase disappears, Cong et al. [35] have observed an abrupt decrease of $T_{m}$ when the Co exceeds $6 \%$ in Ni-rich $\mathrm{Ni}_{2+x-y} \mathrm{Co}_{y} \mathrm{MnGa}_{1-x}(x=0.12,0 \leq y \leq 0.56)$, i.e., $T_{m}$ decreases drastically from $375 \mathrm{~K}$ to $236 \mathrm{~K}$ when the Co content changes from $y=0.24$ to 0.32 . In the alloys with $y \geq 0.4$, no martensitic transformation was observed in the temperature range $188-573 \mathrm{~K}$ in the DSC measurements [35]. A recent study of Ni-rich $\mathrm{Ni}_{2-y} \mathrm{Co}_{y} \mathrm{Mn}_{1+x} \mathrm{Ga}_{x}(x=0.16,0 \leq y \leq 0.4)$ performed by Pushin et al. [44] has shown that upon Co doping (up to $y=0.4$ ), the structural transition temperature is found to decrease from room temperature to 6-10 K. Another interesting point is related to the possibility of inducing a paramagnetic gap in $\mathrm{Ni}_{2-y} \mathrm{Co}_{y} \mathrm{Mn}_{1+x} \mathrm{Ga}_{1-x}(0.2 \leq x \leq 0.28$, $0 \leq y \leq 0.36$ ) $[41,43]$ between weakly magnetically ordered martensite and FM austenite. As a result, a reverse magnetostructural phase transformation appears, which is accompanied by a positive change of $\triangle S$ (called inverse MCE). In addition, the inverse MCE across the magnetostructural transition from FM austenite to PM martensite was also found in quinary $\mathrm{Ni}_{2-y} \mathrm{Co}_{y} \mathrm{Mn}_{1+x} \mathrm{Ga}_{1-x-z} \mathrm{In}_{z}(0.26 \leq x \leq 0.28$, $0.35 \leq y \leq 0.368,0.08 \leq z \leq 0.132)[19,48,49]$. With respect to the sequence of phase transitions in Co doped in $\mathrm{Ni}_{2-y} \mathrm{Co}_{y} \mathrm{Mn}_{1+x} \mathrm{Ga}_{1-x}$, Segui et al. [50] have recently stated that the transformation sequence may depend on the quenched-atomic disorder of austenite i.e., poorly ordered alloys (L2 $2_{1}$ austenite) with a high $e / a$ value show a $A_{P M} \Leftrightarrow M_{P M} \Leftrightarrow M_{F M}$ sequence, whereas disordered alloys (B2 austenite) display the $A_{P M} \Leftrightarrow A_{F M} \Leftrightarrow M_{P M} \Leftrightarrow M_{F M}$ sequence; where $A(M)$ denotes austenite (martensite), respectively. On the other hand, for samples with a low $e / a$ value, the transformation sequence changes from $A_{P M} \Leftrightarrow A_{F M} \Leftrightarrow M_{P M} \Leftrightarrow M_{F M}$ to $A_{P M} \Leftrightarrow A_{F M} \Leftrightarrow M_{F M}$.

In order to understand the influence of $\mathrm{Co}$ addition on the magnetic and structural properties of Ni-Mn-Ga more profoundly, several theoretical studies have been performed using model and first-principles calculations in the framework of density functional theory (DFT) [46,51-54]. Recently, Kanomata et al. [46] have analyzed the phase diagram of $\mathrm{Ni}_{2} \mathrm{MnGa}_{1-y} \mathrm{Co}_{y}(0 \leq y \leq 0.2)$ using a Landau-type free energy expansion in strain and magnetic order parameters. They observed that for optimal model parameters, the theoretical phase diagram reproduces the main features of the experimental phase diagram. On the basis of DFT calculations, Entel et al. [51] have studied the effect of $\mathrm{Ga}$ substitution by $\mathrm{Co}\left(3 \mathrm{at} \%\right.$ ) on the physical properties of $\mathrm{Ni}_{2} \mathrm{MnGa}$, finding that the addition of Co causes an increase of magnetic moments and an increase of the energy difference between austenite and martensite phases. The estimated values of $T_{C}$ and $T_{m}$ were found to increase by 80 and $140 \mathrm{~K}$, respectively.

The effect of $\mathrm{Co}$ content on the magnetic property and phase stability of $\mathrm{Ni}_{2-y} \mathrm{Co}_{y} \mathrm{MnGa}$ $(0 \leq y \leq 0.75)$ alloys has also been investigated by Chang-Long et al. [52]. They show that both the 
phase stability of austenite and the Curie temperature increase with increasing Co content. Similar investigations of the physical properties of $\mathrm{Ni}_{2-y} \mathrm{Co}_{y} \mathrm{MnGa}(0 \leq y \leq 1)$ have been performed by Bai et al. [53]. They observed that the lattice parameter $a$ of austenite decreases, while for martensite, $a$ increases and $c$ decreases with increasing Co content. Besides, the Curie temperature increases with Co content. Detailed ab initio investigations of $\mathrm{Co}$ - and $\mathrm{Cu}$-doped $\mathrm{Ni}_{2} \mathrm{MnGa}$ along the tetragonal deformation path have been recently presented by Zeleny et al. [54] with substitution of Co for Ni and $\mathrm{Cu}$ for $\mathrm{Mn}$ or $\mathrm{Ga}$ with up to $12.5 \%$. These simulations show that Co doping has a much stronger effect on the equilibrium $(c / a)$ value compared to $\mathrm{Cu}$. Moreover, the increase of Co content causes the equilibrium $c / a$ ratio to decrease from $1.25(0 \% \mathrm{Co})$ to $1.16(7.5 \%)$, and a further increase of Co concentration up to $12.5 \%$ destabilizes the martensitic phase. The theoretical investigations widely confirm the experimental trends and properties of the Ni-Mn-Ga-Co alloys.

In this paper, we extend the zero-temperature first-principles calculations of Co-doped Ni-Mn-Ga within the DFT scheme to finite-temperature Monte Carlo (MC) simulations, where exchange coupling constants, magnetic moments, and anisotropy energy are taken from ab initio calculations. This allows us to simulate the temperature dependence of magnetic and thermodynamic properties, as well as the magnetocaloric effect of Ni-Co-Mn-Ga across a magnetostructural phase transformation.

\section{Construction of the Model Hamiltonian}

For the finite-temperature MC simulations of Co-doped Ni-Mn-Ga alloys, we employ the extended Potts-Blume-Emery-Griffiths Hamiltonian, which allows one to describe a system of interacting magnetic moments on a polycrystalline lattice. Each atom of the lattice is characterized by magnetic $S_{i}$ and structural $\sigma_{i}$ degrees of freedoms. Since the magnetic moment of Ga is about several orders of magnitude smaller than the magnetic moments of $\mathrm{Mn}, \mathrm{Ni}$, and $\mathrm{Co}$, we consider only the structural degree of freedom for Ga. We would like to point out that in case of non-stoichiometric $\mathrm{Ni}_{50} \mathrm{Mn}_{25+x} \mathrm{Z}_{25-x}$ $(\mathrm{Z}=\mathrm{Ga}, \mathrm{In}, \mathrm{Sn}, \mathrm{Sb}$ ) Heusler alloys, there are $\mathrm{Mn}$ magnetic moments interacting antiferromagnetically (AFM) with each other. The first $\mathrm{Mn}$ atom, which is referred to as $\mathrm{Mn}_{1}$, occupies the original $\mathrm{Mn}$ sublattice, while the second $\mathrm{Mn}$ atom $\left(\mathrm{Mn}_{2}\right)$ is located on the Ga sublattice. Ab initio calculations reveal strong AFM $\mathrm{Mn}_{1}-\mathrm{Mn}_{2}$ interactions, because of the shorter distance between $\mathrm{Mn}_{1}-\mathrm{Mn}_{2}\left(d=0.5 a_{0}\right)$ compared to $\mathrm{Mn}_{1}-\mathrm{Mn}_{1}$ and $\mathrm{Mn}_{2}-\mathrm{Mn}_{2}$ distances $\left(d=\sqrt{2} / 2 a_{0}\right)$ [55-60,66]. Here, $a_{0}$ is the lattice parameter of the cubic $\mathrm{L} 2{ }_{1}$.

In order to describe approximately the effect of polycrystallinity (or multidomain magnetic states), we divided the three-dimensional simulation cell into 16 domains with random initial spin configurations. For the $q$-state Potts model, the spin configurations correspond to discrete spin states $S_{i} \in\{1,2, \ldots, q\}$, where the maximum number of possible $q$ states depends on a total spin moment $S$ of an atom defined by the $2 S+1$ multiplet. In each magnetic domain, all atoms have the same configuration as the variable $S_{k}$, which defines the 'easy axis' and characterizes the action of magnetic anisotropy in each domain. Similar to the multi-domain Heisenberg model, all spins will be aligned with one another pointing in the same direction as enforced by the magnetic anisotropy. Hence, for the Heisenberg and Potts model, different domain magnetizations will approximately lead to zero total magnetization in a zero or low external magnetic field. 
In this work, our main concern is the $q$-state Potts model in combination with the three state Blume-Emery-Griffiths (BEG) model, which was previously applied to Heusler systems [55,60-65]. Note that the Potts model is suitable for investigations of first-order phase transitions, as well as recrystallization problems, such as grain growth kinetics, grain size distribution and their topologies, etc. We would like to note that most of the formulas have already been published (see, [55,60-65]), but for the sake of completeness and discussion, we briefly outline here the model Hamiltonian. In the Potts Hamiltonian Equation (1), the magnetic exchange interactions, magnetic anisotropy, and externally magnetic fields are specified by:

$$
\mathcal{H}_{m a g}=-\sum_{\langle i j\rangle} J_{i j}^{m} \delta_{S_{i}, S_{j}}+K_{\text {ani }} \sum_{i} \delta_{S_{i}, S_{k}} \mu_{i}^{2}-g \mu_{B} H_{\text {ext }} \sum_{i} \delta_{S_{i}, S_{g}} \mu_{i} .
$$

where $J_{i j}$ are the exchange coupling constants between magnetic moments $\mu_{i}$ at sites $i$ and $j$ of cubic and tetragonal Heusler lattices. $S_{i}$ is the Potts' spin at site $i$, which can take on $q$ integer values depending on the total spin moment $S$ of an atom. The spin moment of Mn is $S=5 / 2$; hence, we associate the $2 S+1$ possible spin projections $\left(-5 / 2,-3 / 2,-1 / 2,1 / 2,3 / 2\right.$ and 5/2) with the $q_{\mathrm{Mn}}=1 \ldots 6$ states. Likewise, we assume $S=1(3 / 2)$ for $\mathrm{Ni}(\mathrm{Co})$, respectively, with the possible $q_{\mathrm{Ni}}=1 \ldots 3\left(q_{\mathrm{Co}}=1 \ldots 4\right)$ states. $K_{a n i}$ is the magnetic anisotropic constant. $H_{e x t}$ is the external magnetic field. $\mu_{B}$ is Bohr's magneton, and $g$ is the Landé factor. The values of $J_{i j}$ and magnetic moments $\mu_{i}$ are obtained from ab initio calculations for austenite and martensite separately. The first Kronecker symbol, $\delta_{S_{i}, S_{j}}$, restricts the spin-spin interactions to the interactions between the same $S_{i}$ states for Mn, Co and Ni. The coupling of the spin $S_{i}$ to the magnetic anisotropy energy $K_{a n i}$ is specified by the second Kronecker symbol $\delta_{S_{i}, S_{k}}$. Here, all spins in a domain have then the same $q$ state as $S_{k} ; S_{k}$ may be chosen to be different from domain to domain. The coupling to the external magnetic field is specified in the third term by $S_{g}$, where we align all spins by choosing $S_{g}=1\left(H_{e x t} \geq 0\right) .\langle i, j\rangle$ denotes here, for the magnetic interactions, a sum over neighbors up to the sixth coordination shell in the Heusler lattice.

The structural part is described by the degenerated three-state Blume-Emery-Griffiths (BEG) model, which allows for a structural transformation from the cubic (austenitic) phase to the tetragonal (martensitic) phase,

$$
\begin{aligned}
\mathcal{H}_{e l}= & -\sum_{\langle i j\rangle} \sigma_{i} \sigma_{j}\left\{J+g \mu_{B} H_{e x t}\left(U_{1} \sum_{i} \delta_{S_{i}, S_{g}}+U_{2} g \mu_{B} H_{e x t}\right)\right\} \\
& -K \sum_{\langle i j\rangle}\left(1-\sigma_{i}^{2}\right)\left(1-\sigma_{j}^{2}\right)-k_{B} T \ln (p) \sum_{i}\left(1-\sigma_{i}^{2}\right),
\end{aligned}
$$

where $J$ and $K$ are the structural coupling constants for the tetragonal and cubic states, respectively. The variable $\sigma_{i}$ defines the deformation state at each lattice site where $\sigma_{i}= \pm 1$ and $\sigma_{i}=0$ specify the tetragonal and cubic phases, respectively. $p$ is the degeneracy factor of the cubic phase that characterizes the number of structural variants. $U_{1}$ and $U_{2}$ are dimensionless magnetoelastic interaction constants. $T$ is the temperature, and $k_{B}$ is the Boltzmann constant. The summation $\langle i, j\rangle$ is taken over nearest neighbor pairs only. We would like to point out that in previous work [60,62-64], we have used a slightly different term $\mathcal{H}_{e l}$ with respect to the action of the external magnetic field, where a shift of the structural transition temperature by the applied magnetic field had been modeled using the previous $\mathcal{H}_{e l}$ Hamiltonian (using 
only the $U_{1}$ parameter) for not too large magnetic fields. However, this is insufficient to model the martensitic transformation in the case of large magnetic fields. Therefore, we added to Equation (2) the second and third terms with dimensionless magnetoelastic constants $U_{1}$ and $U_{2}$. Somewhat similar terms $\left(\eta_{4} e^{2} m \mu_{B} H\right.$ and $\left.\eta_{3} e^{2}\left(\mu_{B} H\right)^{2}\right)$ have been used in a Landau functional theory of elastic and magnetic interactions in [67].

The coupling between the magnetic and structural subsystems is defined in terms of the magnetostructural interaction as:

$$
\left.\mathcal{H}_{i n t}=2 \sum_{\langle i j\rangle} U_{i j} \delta_{S_{i}, S_{j}} \mu_{i} \mu_{j}\left\{\left(\frac{1}{2}-\sigma_{i}^{2}\right)\left(\frac{1}{2}-\sigma_{j}^{2}\right)-\frac{1}{4}\right)\right\}
$$

where $U_{i j}$ are magnetoelastic interaction parameters.

The total Hamiltonian, which is used in the Monte Carlo simulations, is then the sum of all terms,

$$
\mathcal{H}=\mathcal{H}_{\text {mag }}+\mathcal{H}_{\text {el }}+\mathcal{H}_{\text {int }} .
$$

For a halfway realistic description of low-isofield magnetization curves of the magnetocaloric materials as a function of temperature in polycrystalline Heusler alloys, we need to specify intra-domain, as well as inter-domain interactions. This issue is somewhat ambiguous, because magnetic domains are separated by domain walls in ferromagnetic materials by several tens of $\mathrm{nm}$ in width [68], depending on the ratio of $J_{i j}$ and and $K_{a n i}$. Furthermore, one would have to take into account the magnetic dipolar interaction in a finite system in analogy to the interaction between magnetic nanoparticles. On the other hand, the number of atoms in the simulation cell used in the MC simulations is of the order of a few thousand of atoms giving rise to a length scale of $c a .50 \mathrm{~nm}$, which is not much larger than the typical width of magnetic domain walls in metals. Therefore, for the sake of simplicity and computational time saving, we assume that spins belonging to neighboring domains do not interact in a zero magnetic field. Although this is a very crude approximation, it would be helpful to perform the MC simulations with the Potts model. However, for a finite magnetic field, we want to account for the competition of external and anisotropy fields. We use a very simple procedure by choosing a random number $r \in[0,1]$ and compare it with the probability containing the ratio of anisotropy and Zeeman energy,

$$
\mathcal{W}_{d}^{(i)}=\min \left\{1, \exp \left(-\frac{\mu_{i}^{2}\left|K_{\text {ani }}\right|}{g\left|\mu_{i}\right| \mu_{B}\left|H_{\text {ext }}\right|}\right)\right\} .
$$

Such that for $r<\mathcal{W}_{d}$, we take into account both the intra-domain and inter-domain exchange interactions using the $J_{i j}$ coupling constants; otherwise, only the intra-domain interactions are taken into account. Obviously, it follows from Equation (5) that in the case of a magnetic field, when the Zeeman energy is smaller than the magnetic anisotropy energy, $\mathcal{W}_{d}$ becomes small and, effectively, a limited number of atoms will participate in the inter-domain exchange interactions, resulting in a small total magnetization. In the case of a high magnetic field, when the field energy is much larger than the anisotropy energy, $\mathcal{W}_{d}$ approaches one, and all spins will participate in the inter-domain interactions aligning spins along the applied magnetic field. This leads to the disappearance of magnetic domains and to a maximum value of magnetization as a single crystal case.

Magnetic $(m)$ and strain $(\epsilon)$ order parameters of our extended Potts-BEG model are given by:

$$
m_{a}=\frac{1}{N_{a}}\left(\frac{q_{a} N_{\max }^{a}-N_{a}}{q_{a}-1}\right), \epsilon=\frac{1}{N} \sum_{i} \sigma_{i} .
$$


Here, $N_{a}$ is the number of $\mathrm{Ni}$, Co or $\mathrm{Mn}_{1(2)}$ atoms, $N_{\text {max }}^{a}$ is the maximum number of identical magnetic states on the lattice, respectively, and $q_{a}$ is the number of $\mathrm{Ni}$, Co or $\mathrm{Mn}_{1(2)}$ magnetic states. In the case of $\epsilon=0$, the austenitic phase is stable, while $\epsilon=1$ corresponds to the martensitic phase with one martensitic variant.

In principle, in Ni-(Co)-Mn-Ga Heusler alloys, cubic austenite (A) and tetragonal martensite (M) with different magnetic moments can coexist across the martensitic phase transformation. Therefore, in order to estimate the total magnetization, we take into account the volume fractions of both phases, which are related through the formula:

$$
f^{\mathrm{A}}(\%)+f^{\mathrm{M}}(\%)=100 \%
$$

where $f^{\mathrm{A}(\mathrm{M})}$ are the volume fractions of austenite and martensite, respectively. Finally, the total magnetization can be expressed as:

$$
M=M^{\mathrm{A}} f^{\mathrm{A}}+M^{\mathrm{M}} f^{\mathrm{M}}
$$

with:

$$
\begin{gathered}
M^{\mathrm{A}(\mathrm{M})}=2 \mu_{\mathrm{Ni}}^{\mathrm{A}(\mathrm{M})} m_{\mathrm{Ni}}(1-y)+y \mu_{\mathrm{Co}}^{\mathrm{A}(\mathrm{M})} m_{\mathrm{Co}}+\mu_{\mathrm{Mn}_{1}}^{\mathrm{A}(\mathrm{M})} m_{\mathrm{Mn}_{1}}+x \mu_{\mathrm{Mn}_{2}}^{\mathrm{A}(\mathrm{M})} m_{\mathrm{Mn}_{2}}+(1-x) \mu_{\mathrm{Ga}}^{\mathrm{A}(\mathrm{M})} m_{\mathrm{Ga}}, \\
f^{\mathrm{A}}=\frac{1}{N} \sum_{i} \sigma_{i}^{(0)}, \quad f^{\mathrm{M}}=\frac{1}{N} \sum_{i}\left(\sigma_{i}^{(+1)}+\sigma_{i}^{(-1)}\right) .
\end{gathered}
$$

Since the magnetic moment of $\mathrm{Ga}$ is much smaller than magnetic moments of $\mathrm{Mn}, \mathrm{Ni}$ and $\mathrm{Co}$, we can neglect the last term in Equation (9). In the present paper, we consider two types of martensitic variants, which can be characterized by the elongation $\left(\sigma_{i}=+1\right)$ and contraction $\left(\sigma_{i}=-1\right)$ of the cubic lattice $\left(\sigma_{i}=0\right)$ along one of the Cartesian directions during a temperature change.

In order to discuss the MCE of Ni-Mn-Ga-(Co) alloys, we must calculate the temperature dependence of the magnetic part of specific heat $C_{m a g}$ and entropy $S_{m a g}$ and the total specific heat $C=C_{m a g}+C_{l a t}$ with magnetic and lattice contributions. We neglect the electronic part of the specific heat. For the lattice heat, we have used the standard Debye approximation [62].

$$
\begin{aligned}
C_{\text {mag }}\left(T, H_{\text {ext }}\right) & =\frac{1}{k_{B} T^{2}}\left[\left\langle\mathcal{H}^{2}\right\rangle-\langle\mathcal{H}\rangle^{2}\right], \\
S_{\text {mag }}\left(T, H_{\text {ext }}\right) & =\int_{T_{1}}^{T_{2}} d T \frac{C_{\text {mag }}\left(T, H_{\text {ext }}\right)}{T} .
\end{aligned}
$$

The isothermal entropy and adiabatic temperature changes with varying external magnetic field can be obtained from [60,62-65],

$$
\begin{array}{r}
\Delta S_{m a g}\left(T, H_{e x t}\right)=S_{m a g}\left(T, H_{e x t}\right)-S_{m a g}(T, 0), \\
\Delta T_{a d}\left(T, H_{e x t}\right)=-T \frac{\Delta S_{m a g}\left(T, H_{e x t}\right)}{C\left(T, H_{e x t}\right)} .
\end{array}
$$

Here, $S_{\text {mag }}\left(T, H_{\text {ext }}\right)$ and $S_{\text {mag }}(T, 0)$ denote the magnetic entropy in the presence of magnetic field $H_{\text {ext }}$ and in a zero field, respectively. 


\section{Computational Details}

The investigations of magnetic and magnetocaloric properties of $\mathrm{Ni}_{50-y} \mathrm{Co}_{y} \mathrm{Mn}_{30} \mathrm{Ga}_{20}(y=0$ and 5 at\%) and $\mathrm{Ni}_{41} \mathrm{Co}_{9} \mathrm{Mn}_{32} \mathrm{Ga}_{16} \mathrm{In}_{2}$ Heusler alloys have been carried out using electronic structure $a b$ initio calculations and Monte Carlo simulations. In order to evaluate the magnetic exchange coupling constants, magnetic moments, and equilibrium magnetic reference states in Co- and In-doped alloys, we first performed $a b$ initio calculations for the cubic $\mathrm{L} 21_{1}$ structure with lattice parameter $a_{0}=5.85 \AA$ and the tetragonal $\mathrm{L}_{0}$ structure with different tetragonal ratios, $c / a$, assuming that the volume of the unit cell does not practically change with structural distortion. The equilibrium lattice parameter $a_{0}$ was taken from our recent ab initio calculations [66] using the Vienna ab initio simulation package (VASP). In that work, we have done equilibrium energy calculations with tetragonal distortion $c / a$ using 16-atom supercell compositions $\mathrm{Ni}_{8} \mathrm{Mn}_{5} \mathrm{Ga}_{3}$ and $\mathrm{Ni}_{7} \mathrm{Co}_{1} \mathrm{Mn}_{5} \mathrm{Ga}_{3}$ corresponding to $\mathrm{Ni}_{50} \mathrm{Mn}_{31.25} \mathrm{Ga}_{18.75}$ and $\mathrm{Ni}_{43.75} \mathrm{Co}_{6.25} \mathrm{Mn}_{31.25} \mathrm{Ga}_{18.75}$, respectively. Calculations have been carried out for two spin configurations referred to as "ferro" (i.e., all spins are parallel) and "ferri" (the spin of $\mathrm{Mn}_{2}$ on the Ga-lattice is reversed). It has been found that the "ferri" solution is energetically favorable for both alloys, and stable martensite is achieved for $c / a=1.31(c / a=1.28)$ for $\mathrm{Ni}_{8} \mathrm{Mn}_{5} \mathrm{Ga}_{3}$ $\left(\mathrm{Ni}_{7} \mathrm{Co}_{1} \mathrm{Mn}_{5} \mathrm{Ga}_{3}\right)$, respectively [66]. For calculations of the coupling constants, $J_{i j}$, we used lattice parameters and magnetic structures for $\mathrm{L} 2_{1}$ austenite and tetragonal $\mathrm{L} 1_{0}$ martensites corresponding to $c / a=1.31(c / a=1.28)$ for $\mathrm{Ni}_{50} \mathrm{Mn}_{30} \mathrm{Ga}_{20}\left(\mathrm{Ni}_{45} \mathrm{Co}_{5} \mathrm{Mn}_{30} \mathrm{Ga}_{20}\right.$ and $\left.\mathrm{Ni}_{41} \mathrm{Co}_{9} \mathrm{Mn}_{32} \mathrm{Ga}_{16} \mathrm{In}_{2}\right)$, respectively.

The calculation of exchange coupling constants has been performed for "ferro" and "ferri" configurations in austenite and martensite using the SPR-KKR package [69,70], where the Heisenberg coupling constants $J_{i j}$ are determinated by employing Liechtenstein's method [71] and the spin-polarized scalar-relativistic mode and atomic sphere approximation (ASA). Chemical disorder in the off-stoichiometric alloys was treated in the framework of the coherent potential approximation (CPA). The angular momentum expansion was up to $l_{\max }=3$. In the self-consistency cycle and in calculation of $J_{i j}, 4,495 k$ points were generated by a $k$-mesh grid of $\{57,57,57\}$. All calculations were converged to $0.01 \mathrm{mRy}$ of total energy. For the exchange correlation energy, we used the general gradient approximation (GGA) in the form of Perdew, Burke and Ernzerhoff [72].

Subsequently, classical MC simulations were carried out in order to obtain the magnetic and magnetocaloric properties of Co- and In-doped $\mathrm{Ni}-\mathrm{Mn}-\mathrm{Ga}$ alloys as a function of temperature and magnetic field. The magnetic moments and exchange couplings obtained from ab initio calculations are used as input data. The MC simulations have been performed for a large enough simulation cell with periodic boundary conditions using the Metropolis algorithm [62]. Changes of the independent variables $q_{\mathrm{Ni}}, q_{\mathrm{Co}}, q_{\mathrm{Mn}_{1(2)}}$ and $\sigma_{i}$ are accepted or rejected according to the single-site transition probability $W=\min \left\{1, \exp \left(-\Delta \mathcal{H} / k_{B} T\right)\right\}$.

As a time unit, we used one MC step consisting of $N$ attempts to change the variables $q_{\mathrm{Ni}}, q_{\mathrm{Co}}, q_{\mathrm{Mn}_{1(2)}}$ and $\sigma_{i}$. We would like to remind the reader that in the case of multidomain structures, the procedure of acceptation of long-range interactions between spins from neighboring domains with probability $\mathcal{W}_{d}$ (see, Equation (5)) is performed for each MC step before the procedure of acceptance or rejection of independent variables $q_{\mathrm{Ni}}, q_{\mathrm{Co}}, q_{\mathrm{Mn}_{1(2)}}$ and $\sigma_{i}$. A uniform random number $r$ is chosen from the interval $[0,1]$ and compared with the probability $\mathcal{W}_{d}$ : if $r<\mathcal{W}_{d}$, then interactions between randomly 
chosen atoms $i$ and its neighbors from other domains are taken into account. Otherwise, if $r>\mathcal{W}_{d}$, the interactions between atom $i$ and its neighbors located in the same domain are taken into account. For each temperature the properties (internal energy of the system $\langle\mathcal{H}\rangle$ and magnetic $\langle m\rangle$ and structural $\langle\epsilon\rangle$ order parameters) were analyzed allowing $5 \cdot 10^{5} \mathrm{MC}$ steps and $10^{4}$ thermalization steps. For simplicity, we consider only two martensitic variants, i.e., the degeneracy factor $p=2$. Values of spin states (i.e., the $q_{\mathrm{Ni}}, q_{\mathrm{Co}}, q_{\mathrm{Mn}_{1(2)}}$ variables) have been chosen randomly by using a number $r$ with $0 \leq r \leq 1$ and fixing $q_{\mathrm{Ni}}, q_{\mathrm{Co}}, q_{\mathrm{Mn}_{1(2)}}$ according to the scheme: if $0 \leq r \leq l / 3$, then $q_{\mathrm{Ni}}=l, l=1,2,3$, if $0 \leq r \leq k / 4$, then $q_{\mathrm{Co}}=k, k=1, \ldots, 4$, and if $0 \leq r \leq n / 6$, then $q_{\mathrm{Mn}_{1(2)}}=n, n=1, \ldots, 6$.

The simulations have been done for $N=L^{3}$ lattice sites, where $L$ is the number of cubic unit cells with lattice parameter $a_{0}$; here, $L=6$ has been taken. Thus, for stoichiometric $\mathrm{Ni}_{2} \mathrm{MnGa}$, we have used a simulation cell containing 1,098 Mn, 1,099 Ga and 1,728 Ni atoms. For off-stoichiometric $\mathrm{Ni}_{2-y} \mathrm{Co}_{y} \mathrm{Mn}_{1+x} \mathrm{Ga}_{1-x}$ alloys, we assume that fractions of $\mathrm{Mn}_{2}(x)$ and $\mathrm{Co}(y)$ are randomly distributed on the $\mathrm{Ga}$ and $\mathrm{Ni}$ sublattices, respectively, according to the alloy composition. In order to create the multi-domain model lattice, we divided the simulation cell into 16 sublattices (domain blocks) with initial random $q$-Potts variables (i.e., the overall spin configuration in each domain is different). For example, for stoichiometric $\mathrm{Ni}_{2} \mathrm{MnGa}$, this amounts to a total of $68 \mathrm{Mn}$ and $108 \mathrm{Ni}$ atoms in each of the 16 domains. In the case of a zero magnetic field, we assume that only intra-domain interactions are present, while for a finite magnetic field, inter-domain interactions are switched using the statistical factor $\mathcal{W}_{d}$ for the competition of magnetic anisotropy and Zeeman energies.

We would like to note that part of the parameters used in the simulations, like magnetic exchange coupling constants $J_{i j}$ and magnetic moments $\mu_{i}$, have been taken from ab initio calculations, while the other parameters, like structural exchange couplings $J$ and $K$, magnetoelastic interaction constants $U_{i j}$, $U_{1}$ and $U_{2}$ and anisotropy constants $K_{a n i}$, have been used as fit parameters in order to reproduce the experimental martensitic transformation temperature and magnetization behavior in different magnetic fields. It is worth noting that the model values of anisotropy and magnetoelastic interaction constants are close to values obtained from experiments and ab initio calculations for Ni-Mn-Ga [73-75]. The model parameters listed in Table 2 and 3 allow, therefore, reasonable simulations of the MCE. We would like to add that in the case of $\mathrm{Ni}_{41} \mathrm{Co}_{9} \mathrm{Mn}_{32} \mathrm{Ga}_{16} \mathrm{In}_{2}$, the "ferro" spin configuration in austenite is more stable compared with the "ferri" spin configuration, because an increase in Co content leads to an enhancement of ferromagnetism, due to the strong FM exchange interactions between Mn and Co.

Table 2. Model parameters (in meV) for Co- and In-doped Ni-Mn-Ga alloys. Parameters $U_{1}$ and $U_{2}$ are the dimensionless constants.

\begin{tabular}{lcccccccc}
\hline Composition & $\boldsymbol{J}$ & $\boldsymbol{K}$ & $\boldsymbol{U}_{\boldsymbol{i j}}^{\mathrm{A}}$ & $\boldsymbol{U}_{\boldsymbol{i j}}^{\mathrm{M}}$ & $\boldsymbol{U}_{\mathbf{1}}$ & $\boldsymbol{U}_{\mathbf{2}}$ & $\boldsymbol{K}_{\boldsymbol{a n \boldsymbol { i }}}^{\mathrm{A}}$ & $\boldsymbol{K}_{\boldsymbol{a n i}}^{\mathrm{M}}$ \\
\hline $\mathrm{Ni}_{50} \mathrm{Mn}_{30} \mathrm{Ga}_{20}$ & 6.6 & 0.5 & 0.5 & 3 & 0.1 & 0.1 & 0.00001 & 0.01 \\
$\mathrm{Ni}_{45} \mathrm{Co}_{5} \mathrm{Mn}_{30} \mathrm{Ga}_{20}$ & 6.2 & 0.5 & 2 & 0.5 & -0.1 & -0.1 & 0.00002 & 0.02 \\
$\mathrm{Ni}_{41} \mathrm{Co}_{9} \mathrm{Mn}_{32} \mathrm{Ga}_{16} \mathrm{In}_{2}$ & 6.3 & 0.5 & 0.4 & 0.2 & -0.2 & -0.8 & 0.00005 & 0.05 \\
\hline
\end{tabular}


Table 3. Magnetic moments (in $\mu_{B}$ ) of Co- and In-doped Ni-Mn-Ga alloys for different structures obtained from $a b$ initio calculations using the SPR-KKR package [69].

\begin{tabular}{lccccccc}
\hline Composition & $\boldsymbol{\mu}_{\mathrm{Mn}_{\mathbf{1}}}$ & $\boldsymbol{\mu}_{\mathrm{Mn}_{\mathbf{2}}}$ & $\boldsymbol{\mu}_{\mathrm{Ni}}$ & $\boldsymbol{\mu}_{\mathrm{Co}}$ & $\boldsymbol{\mu}_{\mathrm{Ga}}$ & $\boldsymbol{\mu}_{\text {In }}$ & $\boldsymbol{\mu}_{\text {tot }}$ \\
\hline $\mathrm{Ni}_{50} \mathrm{Mn}_{30} \mathrm{Ga}_{20}(c / a=1)$ "ferri" & 3.547 & -3.746 & 0.242 & & -0.077 & 3.219 \\
$\mathrm{Ni}_{50} \mathrm{Mn}_{30} \mathrm{Ga}_{20}(c / a=1.31)$ "ferri" & 3.485 & -3.61 & 0.274 & & -0.075 & & 3.25 \\
$\mathrm{Ni}_{45} \mathrm{Co}_{5} \mathrm{Mn}_{30} \mathrm{Ga}_{20}(c / a=1)$ "ferri" & 3.512 & -3.74 & 0.259 & 0.938 & -0.08 & 3.354 \\
$\mathrm{Ni}_{45} \mathrm{Co}_{5} \mathrm{Mn}_{30} \mathrm{Ga}_{20}(c / a=1.28)$ "ferri" & 3.459 & -3.614 & 0.263 & 0.619 & -0.079 & & 3.268 \\
$\mathrm{Ni}_{41} \mathrm{Co}_{9} \mathrm{Mn}_{32} \mathrm{Ga}_{16} \mathrm{In}_{2}(c / a=1)$ "ferro" & 3.476 & 3.602 & 0.405 & 1.114 & -0.098 & -0.086 & 5.185 \\
$\mathrm{Ni}_{41} \mathrm{Co}_{9} \mathrm{Mn}_{32} \mathrm{Ga}_{16} \mathrm{In}_{2}(c / a=1.28)$ "ferri" & 3.418 & -3.63 & 0.261 & 0.62 & -0.083 & -0.069 & 3.279 \\
\hline
\end{tabular}

\section{Computational Results and Discussion}

\subsection{Results of Ab Initio Calculations}

In order to complete the magnetic characterization of $\mathrm{Ni}_{50-y} \mathrm{Co}_{y} \mathrm{Mn}_{30} \mathrm{Ga}_{20}(y=0$ and 5 at\%) and $\mathrm{Ni}_{41} \mathrm{Co}_{9} \mathrm{Mn}_{32} \mathrm{Ga}_{16} \mathrm{In}_{2}$ Heusler alloys, we now consider the $J_{i j}$ in Figure 2 and the electronic density of states (DOS) in Figure 3 of $\mathrm{L} 2_{1}$ and $\mathrm{L} 1_{0}$ Heusler structures for the two different spin configurations labeled "ferri" and "ferro" solutions. The corresponding $J_{i j}$ have been split into $\mathrm{Mn}_{1(2)}-\mathrm{X}\left(\mathrm{X}=\mathrm{Mn}_{1(2)}\right.$, $\mathrm{Mn}_{2(1)} \mathrm{Ni}$, and $\left.\mathrm{Co}\right)$ and $\mathrm{Co}-\mathrm{Y}(\mathrm{Y}=\mathrm{Co}$ and $\mathrm{Ni})$, respectively, where the index "1" refers to $\mathrm{Mn}$ atoms on the Mn sublattice and index " 2 " to Mn atoms on the Ga sublattice. For the $J_{i j}$ calculations, the SPR-KKR code and CPA [69,70] have been used.

It is obvious from Figure 2 that the exchange coupling constant between neighboring atoms is very sensitive to the inter-atomic distance and drastically changes at crossover from $\mathrm{L} 2{ }_{1}$ austenite to $\mathrm{L} 1_{0}$ martensite. In general, the strong competition between FM and AFM interactions leads to the oscillatory RKKY (Ruderman-Kittel-Kasuya-Yosida)-type of interactions.

With respect to the $J_{i j}$ interactions of austenitic $\mathrm{Ni}_{50} \mathrm{Mn}_{30} \mathrm{Ga}_{20}$ in Figure 2a, we see that the $\mathrm{Mn}_{1}-\mathrm{Mn}_{1}$ interaction in the first coordination shell is weaker compared to the following ones. In the first five coordination shells, the $\mathrm{Mn}_{1}-\mathrm{Mn}_{1}$ interaction is FM, but becomes AFM in the sixth coordination shell. A similar behavior of $\mathrm{Mn}_{1}-\mathrm{Mn}_{1}$ interactions has been observed for non-stoichiometric $\mathrm{Ni}_{2+x} \mathrm{Mn}_{1-x} \mathrm{Ga}$ alloys [56,62]. The $\mathrm{Mn}_{2}-\mathrm{Mn}_{2}$ interaction is AFM in the first coordination shell, otherwise it follows the trend of the $\mathrm{Mn}_{1}-\mathrm{Mn}_{1}$ interaction. For the sixth coordination shell, the $\mathrm{Mn}_{2}-\mathrm{Mn}_{2}$ interaction becomes also AFM. The $\mathrm{Mn}_{1}-\mathrm{Mn}_{2}$ interaction is large with AFM interaction up to the forth coordination shell. The origin of large AFM Mn $-M_{2}$ coupling is related to the shorter distance between $\mathrm{Mn}_{1}$ and $\mathrm{Mn}_{2}$ $\left(d=0.5 a_{0}\right)$ compared to $\mathrm{Mn}_{1(2)}-\mathrm{Mn}_{1(2)}\left(d=\sqrt{2} / 2 a_{0}\right)$. Regarding the $\mathrm{Mn}_{1(2)}$-Ni interaction, it is clearly seen that these interactions are strongest in the first coordination shell and then fall off rapidly, which helps to stabilize the FM order. The individual magnetic moments are listed in Table 3. The large value of $J_{i j}$ between Mn is due to the smaller Mn-Ni distance $\left(d=\sqrt{3} / 4 a_{0}\right)$ in contrast to the indirect $\mathrm{Mn}_{1(2)}-\mathrm{Mn}_{1(2)}$ coupling. 
Figure 2. Ab initio magnetic exchange interactions of $\mathrm{Ni}_{50} \mathrm{Mn}_{30} \mathrm{Ga}_{20}$ for (a) $c / a=1$ (cubic), (b) $c / a=1.31$ (tetragonal), exchange interactions of Co-doped $\mathrm{Ni}_{45} \mathrm{Co}_{5} \mathrm{Mn}_{30} \mathrm{Ga}_{20}$ (c) for $c / a=1$ and (d) $c / a=1.28$ and Co- and In-doped $\mathrm{Ni}_{41} \mathrm{Co}_{9} \mathrm{Mn}_{32} \mathrm{Ga}_{16} \mathrm{In}_{2}$ for $(\mathbf{e}) c / a=1$ and (f) $c / a=1.28$, as a function of the distance $d / a$ between pairs of atoms $i$ and $j$ (in units of the lattice constant $a$ ). $T_{C}^{M C}$ marks the Curie temperatures obtained from MC simulations.
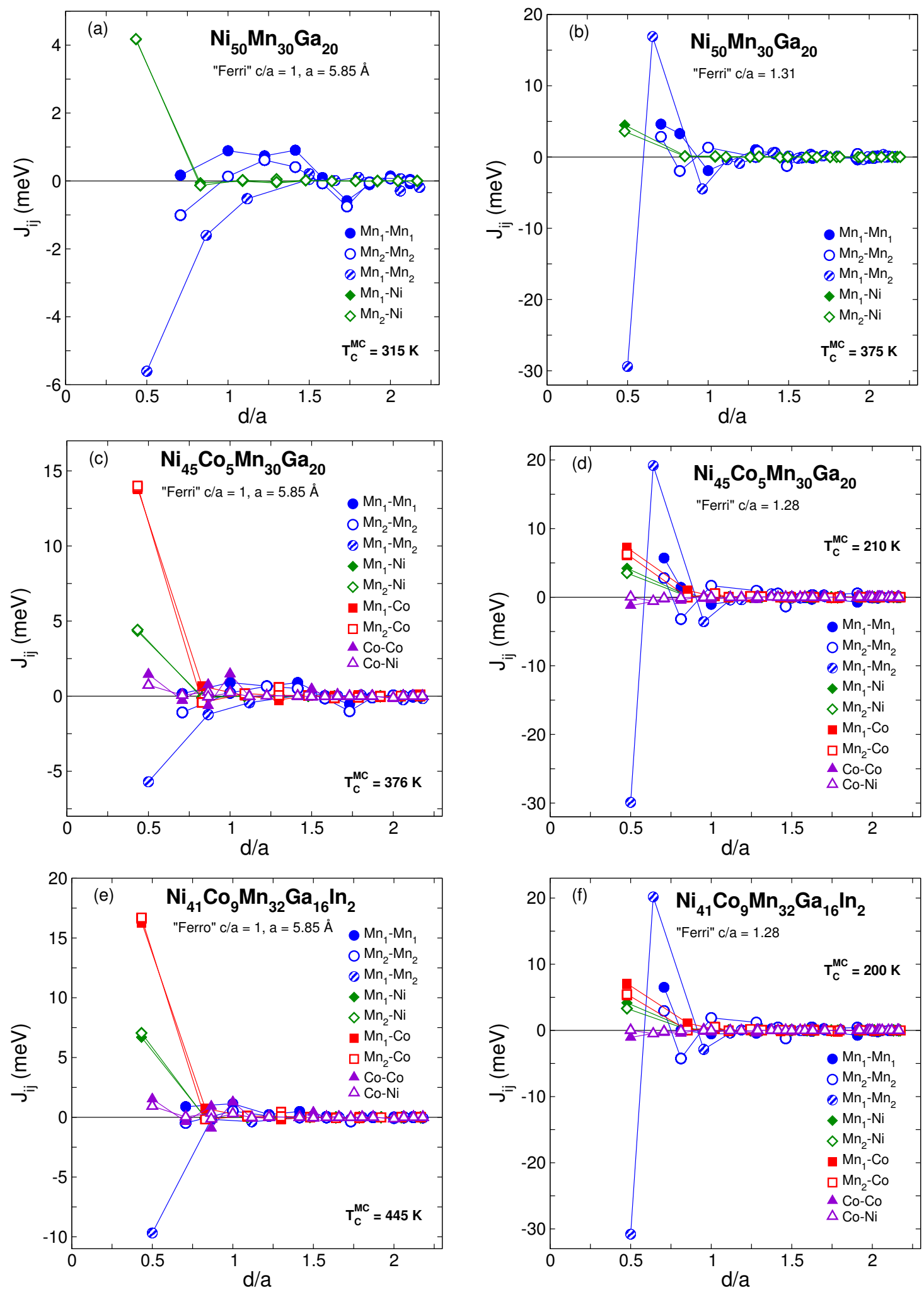
With respect to the exchange interactions in austenite of Co- and In-doped Ni-Mn-Ga alloys (see Figure 2c,e), the behavior of $\mathrm{Mn}_{1(2)}-\mathrm{Mn}_{1(2)}, \mathrm{Mn}_{1}-\mathrm{Mn}_{2}$ and $\mathrm{Mn}_{1(2)}$-Ni interactions is somewhat similar to $\mathrm{L}_{1} \mathrm{Ni}_{50} \mathrm{Mn}_{30} \mathrm{Ga}_{20}$. The strength of $\mathrm{Mn}_{1(2)}-\mathrm{Mn}_{1(2)}$ interactions slightly decreases, while $\mathrm{Mn}_{1(2)}$-Ni coupling constants slightly increase with increasing Co content. For austenite $\mathrm{Ni}_{41} \mathrm{Co}_{9} \mathrm{Mn}_{32} \mathrm{Ga}_{16} \mathrm{In}_{2}$ (see Figure 2e), the nearest neighbor $\mathrm{Mn}_{1}-\mathrm{Mn}_{2}$ coupling constant is larger by one half compared to $\mathrm{Ni}_{50} \mathrm{Mn}_{30} \mathrm{Ga}_{20}$ and $\mathrm{Ni}_{45} \mathrm{Co}_{5} \mathrm{Mn}_{30} \mathrm{Ga}_{20}$. This is because the $J_{i j}$ calculations have been done for the "ferro" spin configuration due to the fact that the FM reference state is stabilized by increasing Co-content. The strongest $\mathrm{Mn}_{1(2)}$-Co interactions are found in austenite of both Co-doped Ni-Mn-Ga alloys, as seen in Figure 2c,e. This is also due to the short distance between Mn and Co atoms (in the case that Co substitutes for $\mathrm{Ni}$ ).

Regarding the exchange coupling constants of martensite $\mathrm{Ni}_{50-y} \mathrm{Co}_{y} \mathrm{Mn}_{30} \mathrm{Ga}_{20}(y=0$ and 5 at $\%)$ and $\mathrm{Ni}_{41} \mathrm{Co}_{9} \mathrm{Mn}_{32} \mathrm{Ga}_{16} \mathrm{In}_{2}$, which are shown in Figure $2 \mathrm{~b}$,d,f, the most striking oscillatory behavior from the FM to AFM interaction is connected with the $\mathrm{Mn}_{1}-\mathrm{Mn}_{2}$ and $\mathrm{Mn}_{1(2)}-\mathrm{Mn}_{1(2)}$ interactions. For instance, for all alloys, the $\mathrm{Mn}_{1}-\mathrm{Mn}_{2}$ interaction in the first coordination shell has a large AFM value of $J_{i j} \approx$ $-30 \mathrm{meV}$, then it changes sign and reaches a large FM value of $J_{i j} \approx 20 \mathrm{meV}$ in the next coordination shell. According to the $\mathrm{Mn}_{1(2)}$-Co interactions within the first coordination shell, $\mathrm{Mn}_{1(2)}$-Ni interactions practically do not change compared to the corresponding interactions for the austenite. The reason for the drastic decrease of the strength in $\mathrm{Mn}_{1(2)}$-Co interactions is related to the slight increase of the $\mathrm{Mn}_{1(2)}$-Co distance due to the martensitic phase transition. A similar behavior of exchange interactions as a function of the distance between atoms in $\mathrm{Ni}_{45} \mathrm{Co}_{5} \mathrm{Mn}_{37} \mathrm{In}_{13}$ has recently been reported by Comtesse et al. [60].

With respect to the different behavior of the individual $J_{i j}$, a detailed discussion has been presented in the literature by Şaşığlu et al. [76] in the context of the Anderson model [77]. The magnetic behavior of the $\mathrm{X}_{2}$ YZ Heusler alloys is governed by three types of interactions: (1) the magnetic $J_{s d}$ interaction between the localized $d$ - and itinerant $s$-like electrons (this includes Coulomb exchange and hybridization); (2) the magnetic $J_{d d}$ exchange interaction resulting from the interaction between localized and itinerant $d$-like electrons; and (3) a superexchange-like interaction through the Z-(sp) electrons. The latter contribution is more important to the indirect exchange mediated mechanism. It is generally accepted that the indirect exchange interaction mediated by the $(s p)$-electrons of the Z-element gives rise to FM order, while the competing superexchange interaction mediated by the same electrons leads to AFM coupling of the Mn-spin moments in the Mn-based Heusler alloys [76,78-83] (although, as stated above, the shorter distance between Mn on its original sublattice and Mn on the Ga sublattice may have AFM contributions from direct magnetic exchange interactions). It should be noted that the Anderson $(s-d)$-impurity model for the formation of localized magnetic moments in a metal includes a hybridization term of strength $V_{\mathbf{k}}$, allowing one to promote electrons between the $s$ - and $d$-states, as well as a local Coulomb term to avoid the double occupancy of the $d$-electron states [77]. Elimination of odd powers of $V_{\mathbf{k}}$ by successive canonical transformations allows one to derive in a systematic fashion conduction-electron-mediated magnetic interactions between the periodically arranged magnetic impurities. In the fourth order of $V_{\mathbf{k}}$ (i.e., eliminating terms of order $V_{\mathbf{k}}$ and $V_{\mathbf{k}}^{3}$ ), the RKKY interaction arises from intermediate states involving low-energy spin excitations corresponding to electron-hole pair formation with a spin-flip transition and a superexchange-type of interaction induced by virtual high-energy charge excitations, which promote electrons from the localized $d$-states to states 
above $E_{F}[76,84,85]$. See Ref. [76] and the original literature [84,85] for a more detailed discussion of these terms. In reciprocal space and at zero temperature, the (strength of) interactions have the following form:

$$
J_{R K K Y}=\sum_{n n^{\prime}, \mathbf{k}}\left\{\frac{\left|V_{n \mathbf{k}}\right|^{2}\left|V_{n^{\prime} \mathbf{k}^{\prime}}\right|^{2}}{\left(\epsilon_{n^{\prime} \mathbf{k}^{\prime}}-\epsilon_{d}\right)^{2}} \frac{\Theta\left(E_{F}-\epsilon_{n \mathbf{k}}\right) \Theta\left(\epsilon_{n^{\prime} \mathbf{k}^{\prime}}-E_{F}\right)}{\epsilon_{n^{\prime} \mathbf{k}^{\prime}}-\epsilon_{n \mathbf{k}}}+c . c .\right\} \mathbf{q}=0 \frac{V^{4} \rho\left(E_{F}\right)}{E_{d}^{2}}
$$

where $\mathbf{k}^{\prime}=\mathbf{k}+\mathbf{q}+\mathbf{G}$ ( $\mathbf{G}$ is a reciprocal lattice vector), $n \mathbf{k}$ labels the band and momentum of the conduction electron Bloch state, $\epsilon_{d}$ is the local energy of the $d$-electron level and $V_{n k}$ is the hybridization matrix element, which couples conduction and localized $d$-electrons (in the spirit of the Anderson model, $\epsilon_{d}^{\uparrow}-\epsilon_{d}^{\downarrow}$ may be assumed to be of the order of the Hubbard $U$-energy [77]).

The $J_{R K K Y}$ interaction is an oscillating function, and its magnitude is mainly determined by the topology of the Fermi surface and may become rather large in the pre-asymptotic region in the case of the Fermi surface nesting; also the DOS at $E_{F}$ determines its size. In the $q=0$ limit, the interaction may be simplified to the limiting value given in Equation (15), where $\rho\left(E_{F}\right)$ is the DOS at $E_{F}$ and $E_{d}$ is the energy required to promote an electron from the $3 d$ level to $E_{F} . V^{4}$ may be considered as an average over the Fermi surface.

In fourth order, $V_{\mathrm{k}}^{4}$, a second term appears, which describes the superexchange interaction given by:

$$
\begin{aligned}
J_{\text {Super-Exch }} & =\sum_{n n^{\prime}, \mathrm{k}}\left\{\frac{\left|V_{n \mathbf{k}}\right|^{2}\left|V_{n^{\prime} \mathbf{k}^{\prime}}\right|^{2}}{\left(\epsilon_{n^{\prime} \mathbf{k}^{\prime}}-\epsilon_{d}\right)^{2}} \frac{\Theta\left(\epsilon_{n \mathbf{k}}-E_{F}\right) \Theta\left(\epsilon_{n^{\prime} \mathbf{k}^{\prime}}-E_{F}\right)}{\epsilon_{n \mathbf{k}}-\epsilon_{d}}+c . c .\right\} \\
\mathbf{q} & \longrightarrow 0 \sum_{n \mathbf{k}} \frac{V^{4}}{\left(E_{F}-\epsilon_{n^{\prime} \mathbf{k}}-E_{d}\right)^{3}}
\end{aligned}
$$

which is more difficult to estimate without explicit numerical work. We would just like to mention that both types of interactions are simultaneously present in the Heusler alloys and contribute to the magnetic interaction. In the case of disordered alloys, we may find nearest neighbor $\mathrm{Mn}$ atoms at shorter interatomic distances, because Mn atoms on the regular Mn-sublattice may be surrounded by Mn on Xand Z-sublattices. This allows for a small overlap of Mn-3d wave functions, which will influence the magnetic interactions in addition to the contributions from Equations (15)-(17).

In this work, we have calculated the $J_{i j}$ by using the alternative method based on Liechtenstein' approach [71] for small rotations of the spin moments with frozen potentials, which expresses the exchange parameters in terms of KKR Green's functions for disordered alloys using the CPA. It is worth noting that the CPA neglects short-range correlations. In order to consider the short-range correlations in disordered alloys, we can take into account the so-called non-local coherent potential approximation (NLCPA). However, so far, this has not really been applied to the Heusler alloys, i.e., a detailed investigation of differences between the CPA and the NLCPA has not really been performed so far in the literature. It appears that the KKR-NLCPA provides a basis for systematically including environmental effects within an $a b$ initio description of disordered alloys. The implementation proposed by Ebert et al. [86] is designed for the treatment of magnetically-ordered alloys. Although there are systematic deviations between NLCPA and CPA, the average NLCPA result, for example for the electronic DOS, is close to that obtained using the standard single-site CPA. The scatter of orbital magnetic moments is greater than for the spin moments. For Heusler alloys with their multi-lattices, it would be interesting to 
apply the NLCPA, which exhibits strong atomic short-range order effects; however, that is beyond the scope of the present paper, and we have only calculated the $J_{i j}$ by using the single-site CPA. However, we would like to point out that in full-Heusler alloys [87], the short-range interactions have a minimal influence on the electronic structure; therefore, the CPA works well. In general, our results for the stoichiometric and cases of $\mathrm{Ni}_{2} \mathrm{Mn}_{1+x}(\mathrm{Ga}, \mathrm{In}, \mathrm{Sn}, \mathrm{Sb})_{1-x}$ (see [55,56,58,59,62]) agree well with those obtained by the frozen-magnon approach to $\mathrm{Ni}_{2} \mathrm{MnZ}(\mathrm{Z}=\mathrm{Ga}$, In, Sn, Sb) [76,78], apart from small differences.

The calculated total and partial DOS for austenitic and martensitic phases of $\mathrm{Ni}_{50} \mathrm{Mn}_{30} \mathrm{Ga}_{20}$ and $\mathrm{Ni}_{45} \mathrm{Co}_{5} \mathrm{Mn}_{30} \mathrm{Ga}_{20}$ alloys are shown in Figure 3. Obviously, substitution of 5 at $\%$ Co for $\mathrm{Ni}$ in $\mathrm{Ni}_{50} \mathrm{Mn}_{30} \mathrm{Ga}_{20}$ does practically not change the general shape of the austenitic and martensitic DOS. The antibonding parts of the DOS around and above the Fermi energy $E_{F}$ have mostly contributions from $\mathrm{Mn}_{1} 3 d$ states, while bonding parts and non-bonding parts have $\mathrm{Mn}_{1}$ and $\mathrm{Ni} 3 d$ contributions. Since the concentrations from $\mathrm{Mn}_{2}$ and Co atoms are small, they contribute little to the total DOS. Nevertheless, in all cases, the small peak in the majority anti-bonding states observed above Fermi $E_{F}$ is caused by contributions from $\mathrm{Mn}_{2} 3 d$ states. It is also found that the contributions from the $\mathrm{Mn}_{1}$ and $\mathrm{Mn}_{2} 3 d$ states to the total DOS are opposite of each other. For the majority DOS of $\mathrm{Mn}_{1}$, the main two peaks below $E_{F}$ are occupied, while for the minority DOS, the antibonding peak is above $E_{F}$. On the other hand, the partial DOS of $\mathrm{Mn}_{2}$ below $E_{F}$ and in the majority DOS above $E_{F}$ clearly have antiparallel alignment of their spin, which lowers the total magnetic moment. With respect to the partial DOS of Ni, we see that the majority and minority spin states are practically symmetrical, which results in the small magnetic moment of Ni. Another interesting point following from Figure 3 is related to the stabilization of the martensitic phase due to the formation of a pseudogap at the Fermi level. Figure 4 shows the change of DOS for the case that Co, In and Sn concentrations have been increased, showing no dramatic effect due to the increase of $s, p$ electrons.

\subsection{Results of Monte Carlo Simulations}

We discuss now the $\mathrm{MC}$ simulations of $\mathrm{Ni}_{50-y} \mathrm{Co}_{y} \mathrm{Mn}_{30} \mathrm{Ga}_{20}(y=0$ and 5 at $\%$ ) and $\mathrm{Ni}_{41} \mathrm{Co}_{9} \mathrm{Mn}_{32} \mathrm{Ga}_{16} \mathrm{In}_{2}$ using the the extended Potts-Blume-Emery-Griffiths Hamiltonian. For $\mathrm{Ni}_{50-y} \mathrm{Co}_{y} \mathrm{Mn}_{30} \mathrm{Ga}_{20}$, we used the magnetic exchange coupling constants $J_{i j}$ calculated for the "ferri" magnetic martensitic and austenitic phases, while for the $\mathrm{Ni}_{41} \mathrm{Co}_{9} \mathrm{Mn}_{32} \mathrm{Ga}_{16} \mathrm{In}_{2}$ composition, we have used the $J_{i j}$ of "ferri" martensite and and "ferro" austenite.

Figure 5 shows that the magnetization and strain order parameter coincide for various magnetic fields, pointing to the existence of a coupled magnetostructural phase transition. For $\mathrm{Ni}_{50} \mathrm{Mn}_{30} \mathrm{Ga}_{20}$, the magnetostructural phase transition in the vicinity of $370 \mathrm{~K}$ characterizes the transition from ferrimagnetic martensite to paramagnetic austenite. According to the MC simulations and suitably chosen model parameters, the Curie temperature of austenite, $T_{C}^{(\mathrm{A})}$, is below the Curie temperature of martensite, $T_{C}^{(\mathrm{M})}$. A similar behavior of magnetization of $\mathrm{Ni}_{50} \mathrm{Mn}_{30} \mathrm{Ga}_{20}$ was found experimentally from isofield magnetic measurements by Fabbrici et al. $[19,41]$. On the other hand, for $\mathrm{Ni}_{45} \mathrm{Co}_{5} \mathrm{Mn}_{30} \mathrm{Ga}_{20}$ and $\mathrm{Ni}_{41} \mathrm{Co}_{9} \mathrm{Mn}_{32} \mathrm{Ga}_{16} \mathrm{In}_{2}$ alloys, the magnetostructural transition at $T_{m} \approx 340 \mathrm{~K}$ goes along with the transition from weakly magnetic martensite or PM martensite to ferromagnetically ordered austenite. 
Figure 3. Element resolved density of states of $\mathrm{Ni}_{50} \mathrm{Mn}_{30} \mathrm{Ga}_{20}$ for (a) $c / a=1$ (cubic), (b) $c / a=1.31$ (tetragonal), $\mathrm{Ni}_{45} \mathrm{Co}_{5} \mathrm{Mn}_{30} \mathrm{Ga}_{20}$ for (c) $c / a=1$ and (d) $c / a=1.28$ and $\mathrm{Ni}_{41} \mathrm{Co}_{9} \mathrm{Mn}_{32} \mathrm{Ga}_{16} \mathrm{In}_{2}$ for (e) $c / a=1$ and (f) $c / a=1.28$. "Ferri" means that the spin of $\mathrm{Mn}$ on the Ga sites is reversed.
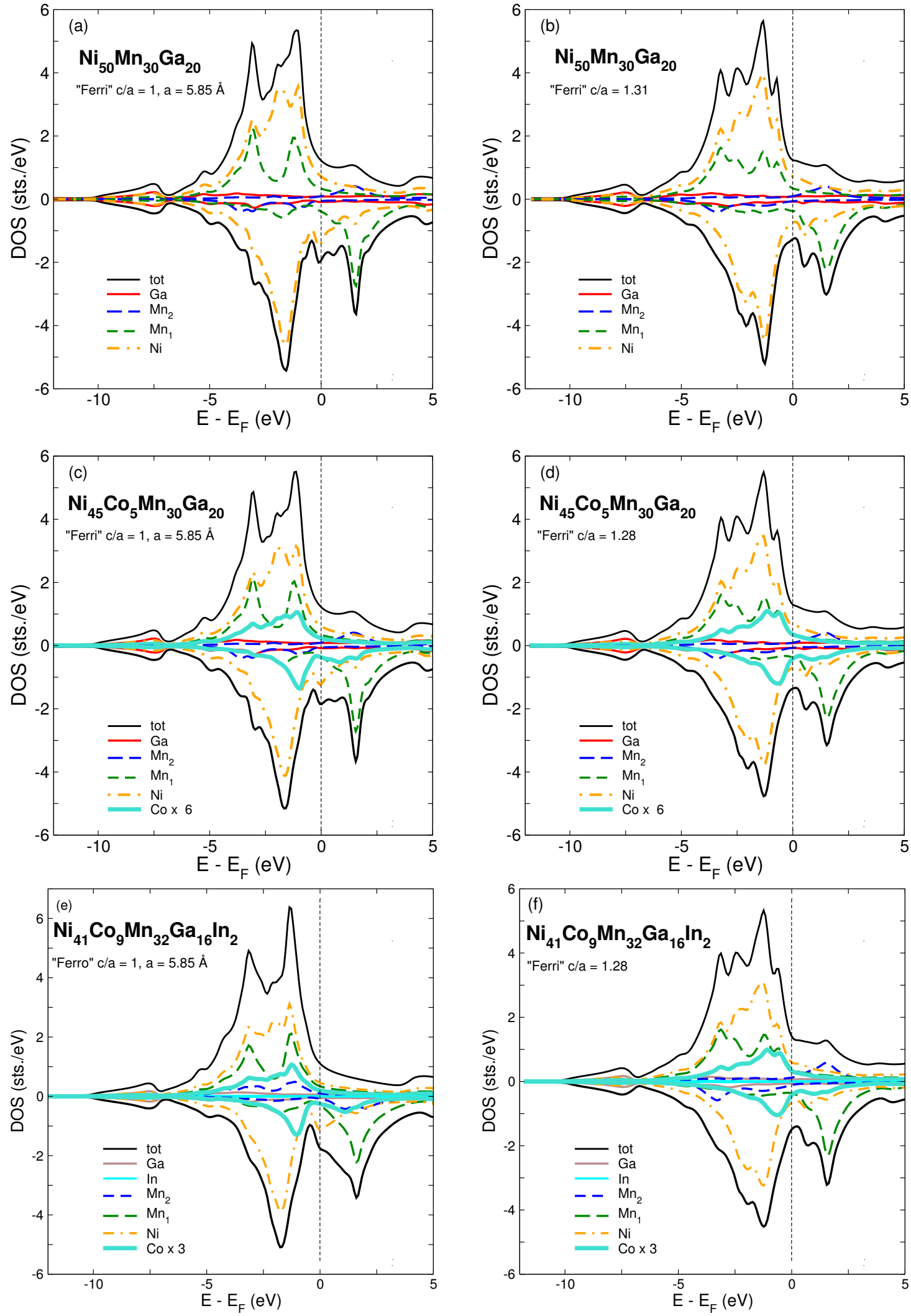
Figure 4. Element resolved density of states of $\mathrm{Ni}_{41} \mathrm{Mn}_{32} \mathrm{Ga}_{9} \mathrm{In}_{9}$ for (a) $c / a=1$ (cubic), (b) $c / a=1.28$ (tetragonal) and $\mathrm{Ni}_{41} \mathrm{Co}_{9} \mathrm{Mn}_{32} \mathrm{Ga}_{9} \mathrm{Sn}_{9}$ for (c) $c / a=1$ and (d) $c / a=1.28$. "Ferri" means that the spin of Mn on the Ga sites is reversed.
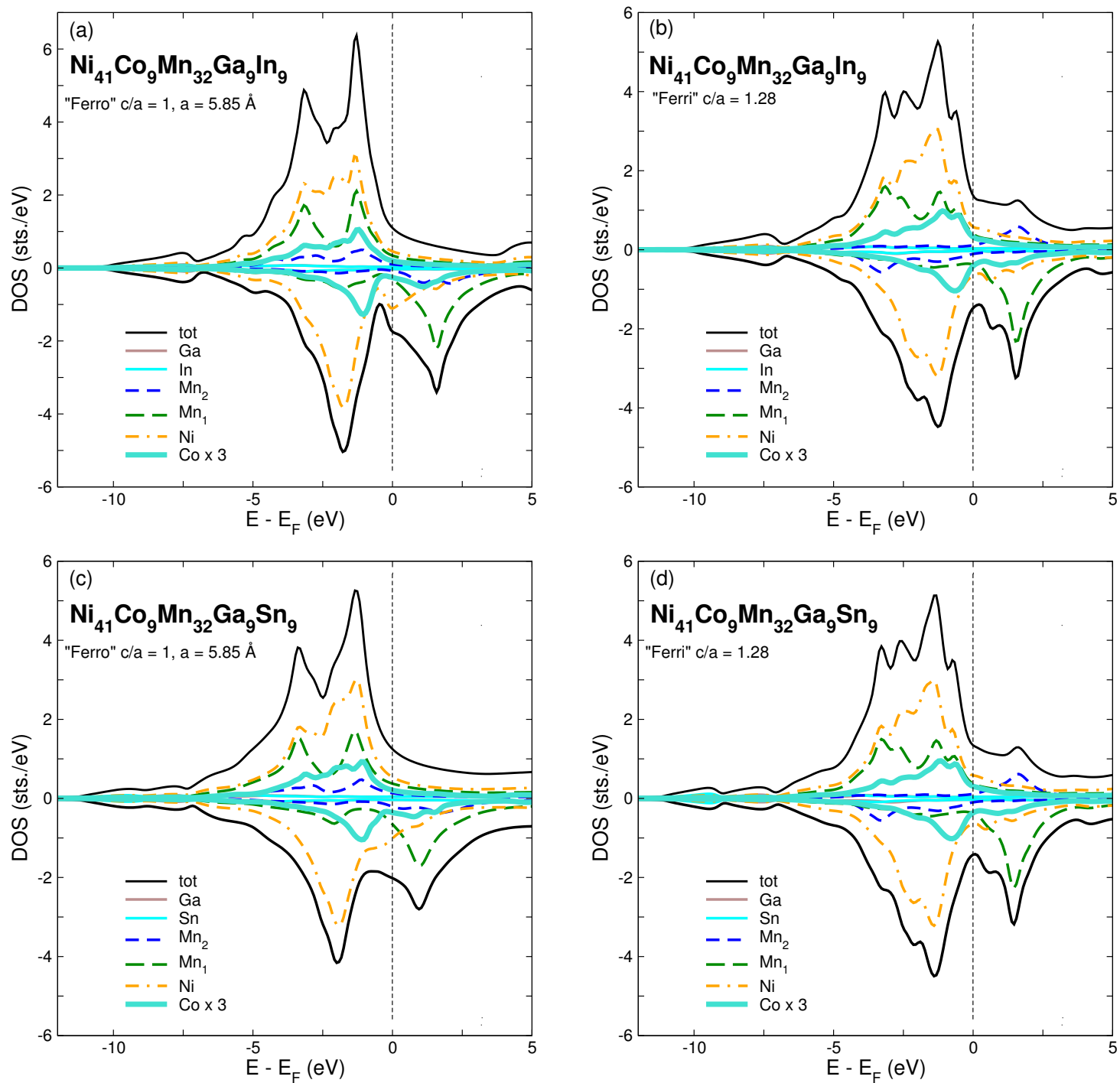

With respect to the Curie temperatures of austenite, these are much larger for Co- and In-doped $\mathrm{Ni}-\mathrm{Mn}-\mathrm{Ga}$ alloys compared to the Curie temperatures of martensite. The insets in Figure 5b,c show the isofield magnetization curves for 0.5 and $2 \mathrm{~T}$ over a larger temperature range. It is obvious from insets that the $T_{C}^{(\mathrm{A})}$ are equal to $\approx 400$ and $445 \mathrm{~K}$ for $\mathrm{Ni}_{45} \mathrm{Co}_{5} \mathrm{Mn}_{30} \mathrm{Ga}_{20}$ and $\mathrm{Ni}_{41} \mathrm{Co}_{9} \mathrm{Mn}_{32} \mathrm{Ga}_{16} \mathrm{In}_{2}$ alloys, respectively. Note that these Curie values are close to the experimental data [19,41]. Concerning the Curie temperature of martensite, an increase of Co content results in a decrease of $T_{C}^{(\mathrm{M})}$. Hence, the paramagnetic gap observed in the vicinity of the structural phase transition is accompanied by an abrupt jump in the magnetization. Note that for $\mathrm{Ni}_{50} \mathrm{Mn}_{30} \mathrm{Ga}_{20}$, the shift of $T_{m}$ by the magnetic field is positive, while for Co- and In-doped Ni-Mn-Ga alloys, the $d T_{m} / d H_{\text {ext }}$ is negative. Moreover, $d T_{m} / d H_{e x t}$ increases with increasing Co content. 
Figure 5. The temperature dependence of calculated magnetization and strain order parameters of (a) $\mathrm{Ni}_{50} \mathrm{Mn}_{30} \mathrm{Ga}_{20}$, (b) $\mathrm{Ni}_{45} \mathrm{Co}_{5} \mathrm{Mn}_{30} \mathrm{Ga}_{20}$ and (c) $\mathrm{Ni}_{41} \mathrm{Co}_{9} \mathrm{Mn}_{32} \mathrm{Ga}_{16} \mathrm{In}_{2}$ alloys for various magnetic fields. The insets show the isofield magnetization curves of $\mathrm{Ni}_{45} \mathrm{Co}_{5} \mathrm{Mn}_{30} \mathrm{Ga}_{20}$ and $\mathrm{Ni}_{41} \mathrm{Co}_{9} \mathrm{Mn}_{32} \mathrm{Ga}_{16} \mathrm{In}_{2}$ for 0.5 and $2 \mathrm{~T}$ calculated to a greater temperature range.
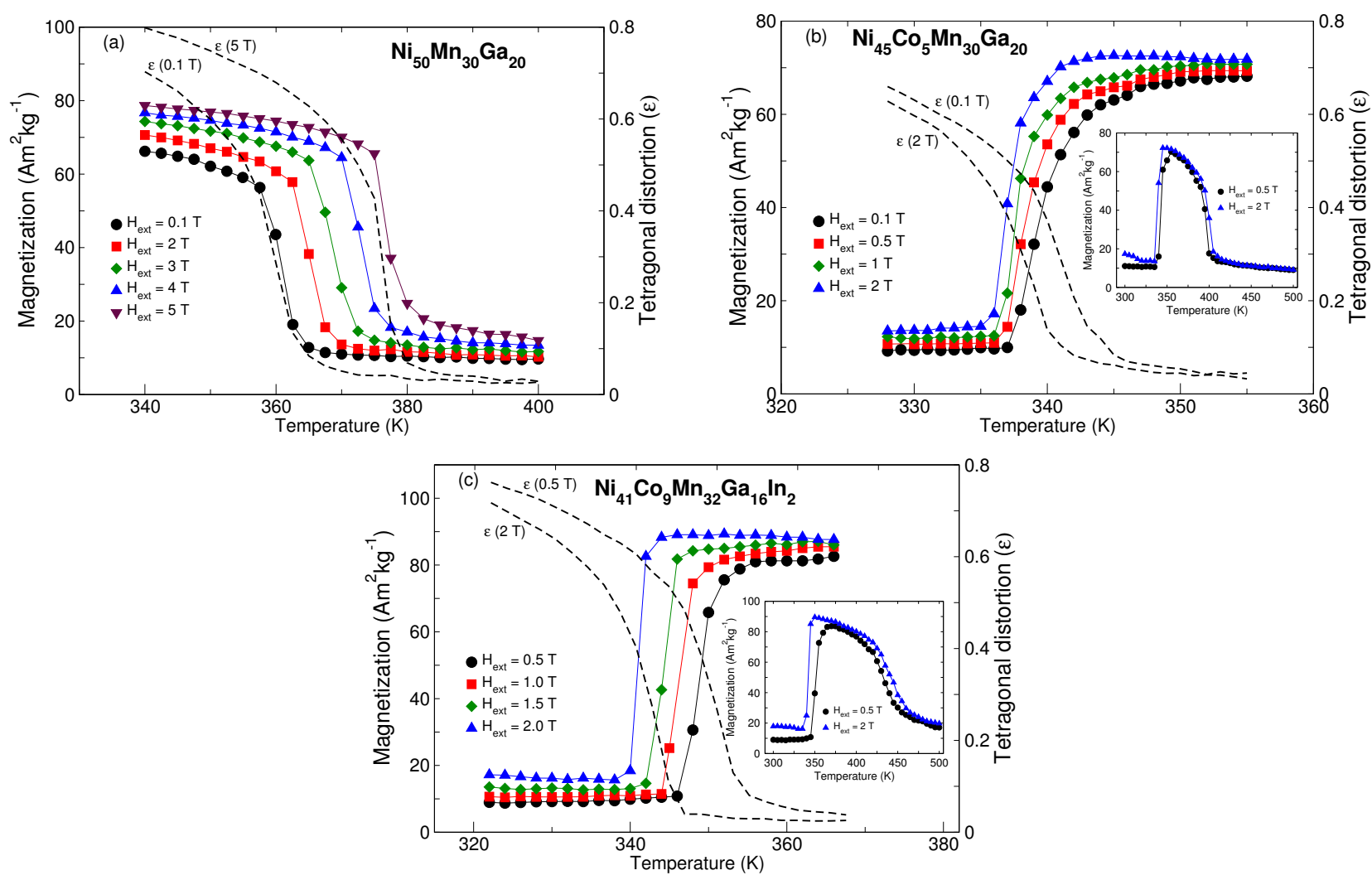

Concerning the change of magnetization at low temperatures, we would like to note that the Bloch law of spin waves is not obtained for the magnetization in the Monte Carlo simulation [88]. This is a well known result known from spin-fluctuation theories, where the magnetization at low temperatures decays in a linear manner with temperature. However, from the diagonalization of the matrix of the $a b$ initio exchange coupling constants, we may obtain, in a mean-field-like fashion, the excitation spectrum (magnon spectrum). This has been done for stoichiometric $\mathrm{Ni}_{2} \mathrm{MnGa}$, but not systematically, in order to incorporate the influence of chemical disorder. Therefore, this has not been included in the paper. Therefore, the isofield-magnetization curves have not been corrected by retaining collective magnetic excitations.

In Figure 6, we present the theoretical temperature dependence of the total specific heat of $\mathrm{Ni}_{50-y} \mathrm{Co}_{y} \mathrm{Mn}_{30} \mathrm{Ga}_{20}$ ( $y=0$ and 5 at $\%$ ) and $\mathrm{Ni}_{41} \mathrm{Co}_{9} \mathrm{Mn}_{32} \mathrm{Ga}_{16} \mathrm{In}_{2}$ in various magnetic fields. The variation of the specific heat curves is shown in the vicinity of the magnetostructural phase transition. These figures show clearly that in the case of $\mathrm{Ni}_{50} \mathrm{Mn}_{30} \mathrm{Ga}_{20},\left(\mathrm{Ni}_{45} \mathrm{Co}_{5} \mathrm{Mn}_{30} \mathrm{Ga}_{20}\right.$ and $\mathrm{Ni}_{41} \mathrm{Co}_{9} \mathrm{Mn}_{32} \mathrm{Ga}_{16} \mathrm{In}_{2}$ ), the peaks of specific heat curves are shifted towards higher (lower) temperature when the magnetic field is applied, respectively. For Co- and In-doped alloys, this is a feature of the metamagnetic phase transition from FM to an AFM or PM state upon cooling, which is associated with the magnetostructural phase transition from ordered ferromagnetic austenite to weakly ordered or PM 
martensite. A comparison of the computational results for the specific heat available from experiments leads to the following observation. The experimental maximal values of specific heat at the $T_{m}$ of $\mathrm{Ni}_{50} \mathrm{Mn}_{30} \mathrm{Ga}_{20}, \mathrm{Ni}_{45} \mathrm{Co}_{5} \mathrm{Mn}_{30} \mathrm{Ga}_{20}$ and $\mathrm{Ni}_{41} \mathrm{Co}_{9} \mathrm{Mn}_{32} \mathrm{Ga}_{16} \mathrm{In}_{2}$ alloys, which are taken for a zero field, are approximately close to $1,750 \mathrm{~J} /(\mathrm{kg} \mathrm{K}), 1,600 \mathrm{~J} /(\mathrm{kg} \mathrm{K})$ and $750 \mathrm{~J} /(\mathrm{kg} \mathrm{K})$, respectively [19]. Our theoretical values for peaks of specific heat for $\mathrm{Ni}_{50} \mathrm{Mn}_{30} \mathrm{Ga}_{20}, \mathrm{Ni}_{45} \mathrm{Co}_{5} \mathrm{Mn}_{30} \mathrm{Ga}_{20}$ and $\mathrm{Ni}_{41} \mathrm{Co}_{9} \mathrm{Mn}_{32} \mathrm{Ga}_{16} \mathrm{In}_{2}$ are $1,720 \mathrm{~J} /(\mathrm{kg} \mathrm{K}), 1,640 \mathrm{~J} /(\mathrm{kg} \mathrm{K})$ and $1,610 \mathrm{~J} /(\mathrm{kg} \mathrm{K})$, respectively. In general, the theoretical specific heat curves are in qualitative agreement with the experimental data. The slight difference in specific heat values for the In-doped sample can be explained by the abrupt change in the theoretical magnetization across the martensitic transformation in comparison with experimental magnetization behavior, which was more smoothly changed at $T_{m}[19]$.

Figure 6. The temperature dependence of total specific heat in the vicinity of the structural phase transformation of (a) $\mathrm{Ni}_{50} \mathrm{Mn}_{30} \mathrm{Ga}_{20}$, (b) $\mathrm{Ni}_{45} \mathrm{Co}_{5} \mathrm{Mn}_{30} \mathrm{Ga}_{20}$ and (c) $\mathrm{Ni}_{41} \mathrm{Co}_{9} \mathrm{Mn}_{32} \mathrm{Ga}_{16} \mathrm{In}_{2}$ alloys for various magnetic fields.
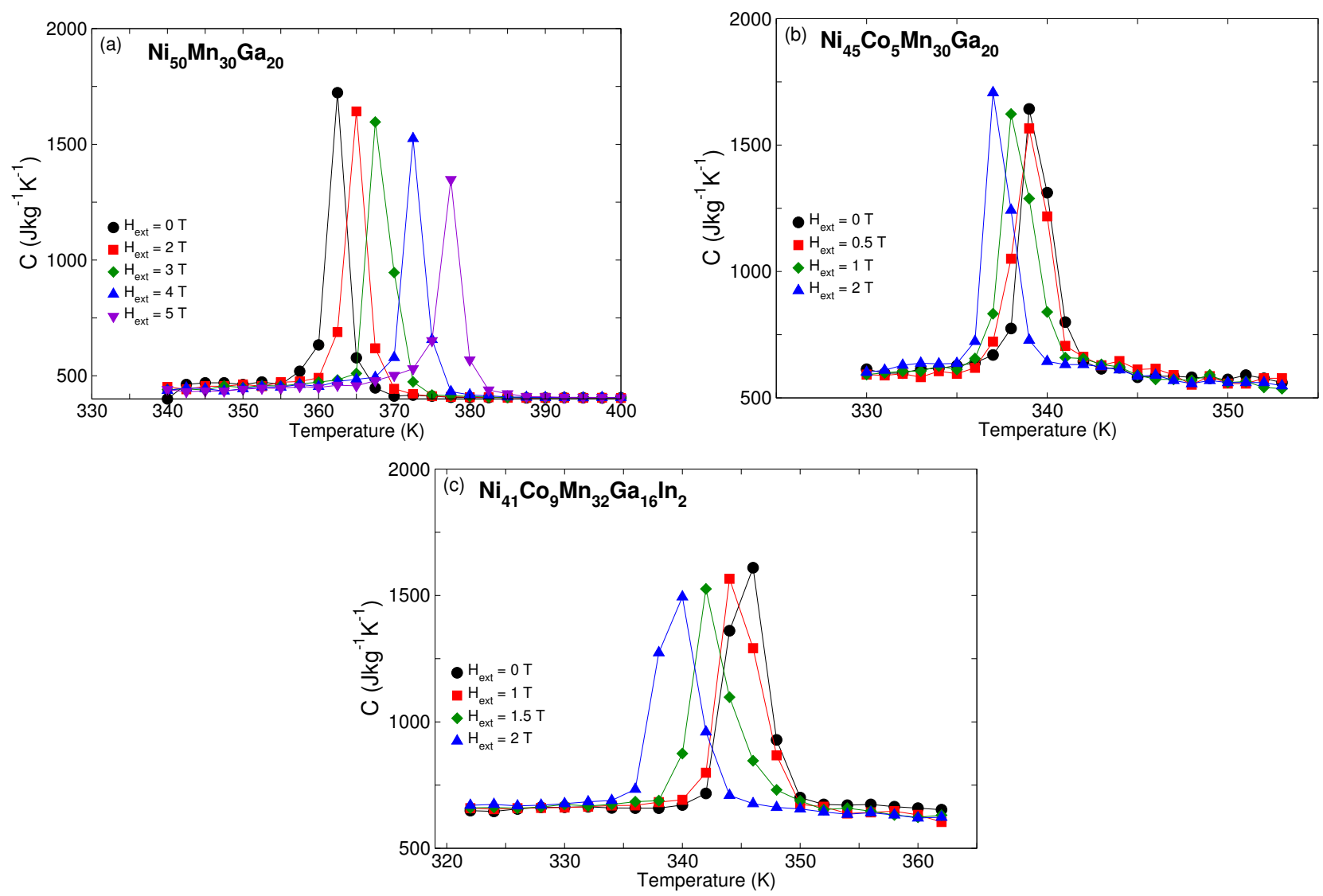

The results for the magnetic entropy of $\mathrm{Ni}_{50} \mathrm{Mn}_{30} \mathrm{Ga}_{20}, \mathrm{Ni}_{45} \mathrm{Co}_{5} \mathrm{Mn}_{30} \mathrm{Ga}_{20}$, and $\mathrm{Ni}_{41} \mathrm{Co}_{9} \mathrm{Mn}_{32} \mathrm{Ga}_{16} \mathrm{In}_{2}$ alloys in magnetic fields of 0 and $2 \mathrm{~T}$ are shown in Figure 7. The curves have been obtained by integrating corresponding specific heat curves using Equation (12). We notice that for $\mathrm{Ni}_{50} \mathrm{Mn}_{30} \mathrm{Ga}_{20}$, the magnetic part of entropy decreases in a magnetic field of $2 \mathrm{~T}$ compared to the entropy in a zero field at the same temperature. Besides, the entropy curve is shifted towards a higher temperature by the magnetic field. This feature is typical for magnetic materials showing an FM-PM transition. In contrast, for Co- and In-doped alloys, the entropies in a magnetic field are larger than the entropies in the zero field in the 
vicinity of the magnetostructural phase transition. Moreover, a magnetic field shifts the entropy curve towards a lower temperature. This points to a metamagnetic phase transition.

Figure 7. The temperature dependence of the isothermal magnetic entropy calculated for $\mathrm{Ni}_{50-y} \mathrm{Co}_{y} \mathrm{Mn}_{30} \mathrm{Ga}_{20}(y=0$ and 5 at\% $)$ and $\mathrm{Ni}_{41} \mathrm{Co}_{9} \mathrm{Mn}_{32} \mathrm{Ga}_{16} \mathrm{In}_{2}$ alloys in magnetic fields of 0 and $2 \mathrm{~T}$.

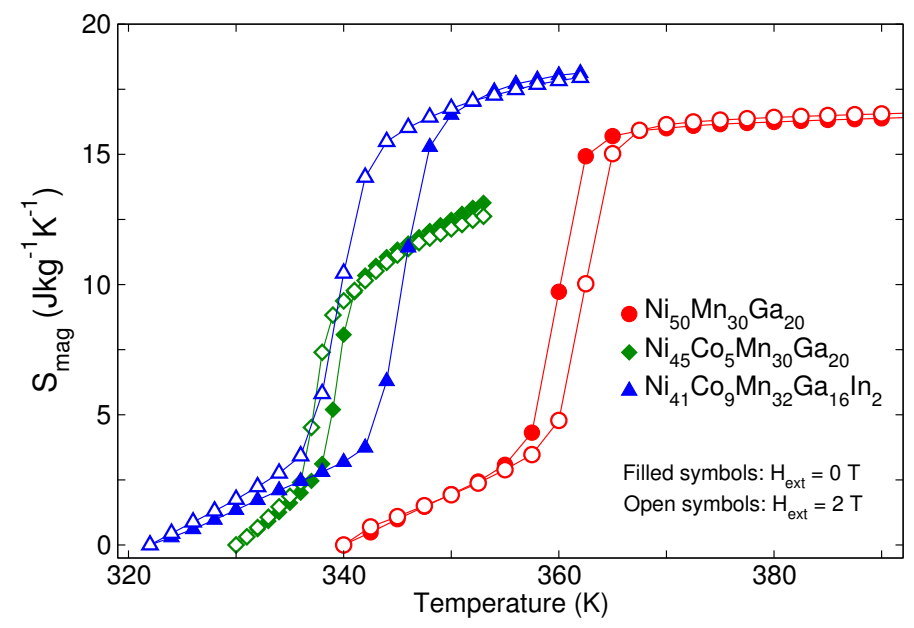

Figure 8. The temperature dependence of (a) isothermal magnetic entropy change and (b) adiabatic temperature change for $\mathrm{Ni}_{50-y} \mathrm{Co}_{y} \mathrm{Mn}_{30} \mathrm{Ga}_{20}(y=0$ and 5 at\%) and $\mathrm{Ni}_{41} \mathrm{Co}_{9} \mathrm{Mn}_{32} \mathrm{Ga}_{16} \mathrm{In}_{2}$ alloys upon variation of the magnetic field from 0 to $2 \mathrm{~T}$. Experimental magnetocaloric curves are shown by dashed lines with open symbols and were measured in a magnetic field change $\Delta H_{e x t}$ of $1.8 \mathrm{~T}$. Experimental data for $\mathrm{Ni}_{50-y} \mathrm{Co}_{y} \mathrm{Mn}_{30} \mathrm{Ga}_{20}(y=0$ and 5 at\%) have been taken from [19], while for the $\mathrm{Ni}_{41} \mathrm{Co}_{9} \mathrm{Mn}_{32} \mathrm{Ga}_{16} \mathrm{In}_{2}$ alloy, the data have been taken from [48].
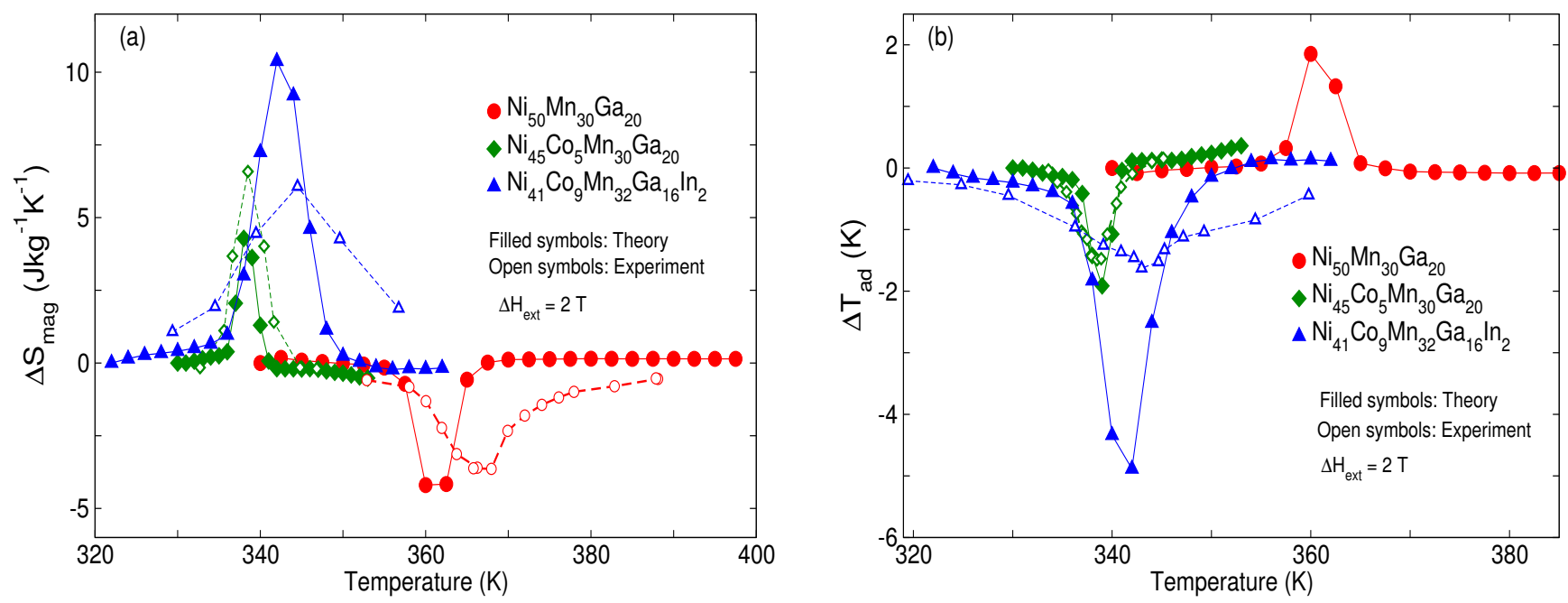

The final computational results for the MCE, isothermal magnetic entropy change $\Delta S_{m a g}$ and adiabatic temperature change $\Delta T_{a d}$ as a function of temperature and magnetic field change from 0 to $2 \mathrm{~T}$ are shown in Figure 8 for $\mathrm{Ni}_{45} \mathrm{Co}_{5} \mathrm{Mn}_{30} \mathrm{Ga}_{20}$ and $\mathrm{Ni}_{41} \mathrm{Co}_{9} \mathrm{Mn}_{32} \mathrm{Ga}_{16} \mathrm{In}_{2}$. In the case of $\mathrm{Ni}_{50} \mathrm{Mn}_{30} \mathrm{Ga}_{20}$, the value of $\Delta S_{m a g}$ is negative, while the value of $\Delta T_{a d}$ is positive at the transition temperature. This 
is a feature of the conventional MCE related to the coupled magnetostructural phase transition from magnetically ordered martensite to magnetically disordered austenite. In contrast, for Co- and In-doped alloys, we observe the inverse MCE characterized by $\Delta T_{a d}<0$ and $\Delta S_{m a g}>0$. In order to compare the theoretical values of MCE with experimental ones [19], we have also inserted the experimental MCE curves by dashed lines with open symbols in Figure 8. It is obvious from Figure 8 that there is a qualitative agreement between the theoretical results obtained in the framework of $a b$ initio calculations and MC simulations using the Potts-BEG model and the experimental data. The slight difference in MCE values between theoretical and experimental results can be attributed to the abrupt change in the theoretical magnetization curves across the martensitic transformation compared with the 'softer' experimental magnetization curves, which change smoothly across $T_{m}[19,48]$.

\section{Conclusions}

In this paper, we presented a theoretical study of the magnetic and magnetocaloric properties of Coand In-doped Ni-Mn-Ga alloys by combining ab initio calculations and Monte Carlo simulations. In the first step, we have done the first-principles calculations of magnetic exchange coupling constants and DOS of $\mathrm{Ni}_{50-y} \mathrm{Co}_{y} \mathrm{Mn}_{30} \mathrm{Ga}_{20}$ ( $y=0$ and 5 at\%) and $\mathrm{Ni}_{41} \mathrm{Co}_{9} \mathrm{Mn}_{32} \mathrm{Ga}_{16} \mathrm{In}_{2}$ alloys for different structural and magnetic configurations. Our previous results have shown that ferrimagnetic order is favorable for both austenitic and martensitic phases in $\mathrm{Ni}_{50-y} \mathrm{Co}_{y} \mathrm{Mn}_{30} \mathrm{Ga}_{20}$, while for $\mathrm{Ni}_{41} \mathrm{Co}_{9} \mathrm{Mn}_{32} \mathrm{Ga}_{16} \mathrm{In}_{2}$, the ferromagnetic reference state in austenite and the ferrimagnetic reference state in martensite are favorable. For all alloys, we have found the strong competition of FM and AFM exchange interactions in the martensitic phase. The addition of Co leads to very large FM interactions between Mn and Co in the austenite phase, whereas for the martensite phase, these interactions are smaller by half those for austenite. In the second step, in order to obtain the finite temperature dependence of the magnetic and magnetocaloric properties of $\mathrm{Ni}-(\mathrm{Co})-\mathrm{Mn}-\mathrm{Ga}-(\mathrm{In})$ alloys, we have used the values of exchange couplings and magnetic moments as input parameters for the microscopic Potts-BEG Hamiltonian. As mentioned before, the addition of $\mathrm{Co}$ to Ni-Mn-Ga alloys results in the splitting of coupled phase transitions at the Curie temperature. As a result, the paramagnetic gap in the vicinity of the martensitic transformation temperature and the metamagnetic behavior of magnetization across the martensitic transformation are observed. Concerning the Curie temperatures, $T_{C}^{\mathrm{A}}$ is found to increase, whereas $T_{C}^{\mathrm{M}}$ decreased with increasing Co content. This behavior can be attributed to the stronger Co-Mn, Mn-Mn and Mn-Ni exchange couplings in austenite compared to the weaker ones in martensite. Moreover, the crossover from a direct to inverse magnetocaloric effect in $\mathrm{Ni}-\mathrm{Mn}-\mathrm{Ga}$ is achieved due to the substitution of $\mathrm{Ni}$ by Co, resulting in the appearance of a paramagnetic gap in martensite. Simulation of the magnetic and magnetocaloric properties of Co-doped Ni-Mn-Ga are in a good qualitative agreement with the available experimental data.

\section{Acknowledgments}

This work is supported by Russian Foundation Basic Research (RFBR) Grant No. 14-02-01085, Russian Science Foundation (RSF) No. 14-12-00570\14 (Section 2), Ministry of Education and Science of Russian Federation (RF) No 3.2021.2014/K (Sections 3 and 4) and the Creation and 
Development Program of National University of Science and Technology (NUST) "MISiS". Peter Entel and Anna Grünebohm acknowledge Deutsche Forschungsgemeinschaft (DFG) (Schwerpunktsprogramm (SPP) 1599) for financial support.

\section{Author Contributions}

Vladimir Sokolovkiy and Peter Entel wrote the manuscript. Vasiliy Buchelnikov, Anna Grünebohm, and Vladimir Sokolovskiy performed the ab initio calculations. Vladimir Sokolovskiy performed the Monte Carlo simulations. All authors have read and approved the final manuscript.

\section{Conflicts of Interest}

The authors declare no conflict of interest.

\section{References}

1. Vasiliev, A.N.; Buchelnikov, V.D.; Takagi, T.; Khovailo, V.V.; Estrin, E.I. Shape-memory ferromagnets. Phys.-Uspekhi 2003, 46, 559-588.

2. Planes, A.; Mañosa, L. Ferromagnetic shape-memory alloys. Mater. Sci. Forum 2006, 512, 145-152.

3. Buchelnikov, V.D.; Vasiliev, A.N.; Koledov, V.V.; Taskaev, S.V.; Khovaylo, V.V.; Shavrov, V.G. Magnetic shape-memory alloys: Phase transitions and functional properties. Phys.-Uspekhi 2006, 49, 871-877.

4. Entel, P.; Buchelnikov, V.D.; Gruner, M.E.; Hucht, A.; Khovailo, V.V.; Nayak, S.K.; Zayak, A.T. Shape Memory Alloys: A Summary of Recent Achievements. Mater. Sci. Forum 2008, 583, $21-41$.

5. Planes, A.; Mañosa, L.; Acet, M. Magnetocaloric effect and its relation to shape-memory properties in ferromagnetic Heusler alloys. J. Phys. Condens. Matter 2009, 21, doi:10.1088/ 0953-8984/21/23/233201.

6. Martynov, V.V.; Kokorin, V.V. The crystal structure of thermally- and stress-induced martensites in $\mathrm{Ni}_{2} \mathrm{MnGa}$ single crystals. J. Phys. III 1992, 2, 739-749.

7. Chernenko, V.A.; Amengual, A.; Cesari, E.; Kokorin, V.V.; Zasimchuk, I.K. Thermal and magnetic properties of stress-induced martensites in Ni-Mn-Ga alloys. J. Phys. IV 1995, 5, doi:10.1051/jp4:1995214.

8. Ullakko, K.; Huang, J.K.; Kantner, C.; O’Handley, R.C. Large magnetic-field-induced strains in $\mathrm{Ni}_{2} \mathrm{MnGa}$ single crystals. Appl. Phys. Lett. 1996, 10, 1966-1968.

9. Murray, S.J.; Marioni, M.A.; Allen, S.M.; O’Handley, R.C.; Lograsso, T.A. 6\% magnetic-field-induced strain by twin-boundary motion in ferromagnetic Ni-Mn-Ga. Appl. Phys. Lett. 2000, 77, 886-888.

10. Sozinov, A.; Likhachev, A.; Lanska, N.; Ullakko, K. Giant magnetic-field-induced strain in NiMnGa seven-layered martensitic phase. Appl. Phys. Lett. 2002, 80, 1746-1748.

11. Sozinov, A.; Likhachev, A.A.; Lanska, N.; Ullakko, K.; Lindroos, V.K. $10 \%$ magnetic-field-induced strain in Ni-Mn-Ga seven-layered martensite. J. Phys. IV 2003, $112,955-958$. 
12. Karaca, H.E.; Karaman, I.; Basaran, B.; Chumlyakov, Y.I.; Maier, H.J. Magnetic field and stress induced martensite reorientation in NiMnGa ferromagnetic shape memory alloy single crystal. Acta Mater. 2006, 54, 233-245.

13. Martynov, V.V.; Kokorin, V.V. Shape memory and multistage superelasticity in Heusler-type Ni-Mn-Ga single crystals. Trans. Mater. Res. Soc. Jpn. 1994, 18B, 839-844.

14. Chernenko, V.A.; L'vov, V.; Pons, J.; Cesari, E. Superelasticity in high-temperature Ni-Mn-Ga alloys. J. Appl. Phys. 2003, 93, 2394-2399.

15. L'vov, V.A.; Rudenko, A.A.; Chernenko, V.A.; Cesari, E.; Pons, J.; Kanomata, T. Stress-induced martensitic transformation and superelasticity of alloys: Experiment and theory. Mater. Trans. 2006, 46, 790-797.

16. Aliev, A.; Batdalov, A.; Bosko, S.; Buchelnikov, V.D.; Dikshtein, I.; Khovailo, V.; Koledov, V.; Levetin, R.; Shavrov, V.; Takagi, T. Magnetocaloric effect and magnetization in Ni-Mn-Ga Heusler alloy in the vicinity of magnetostructural transition. J. Magn. Magn. Mater. 2004, 272, 2040-2042.

17. Albertini, F.; Paoluzi, A.; Pareti, L.; Solzi, M.; Villa, E.; Besseghini, S.; Passaretti, F. Phase transition and magnetocaloric entropy change in Mn-rich $\mathrm{Ni}_{2} \mathrm{MnGa}$ alloys. J. Appl. Phys. 2006, 100, doi:10.1063/1.2218470.

18. Khovaylo, V.V.; Skokov, K.P.; Koshkid'ko, Y.S.; Koledov, V.V.; Shavrov, V.G.; Buchelnikov, V.D.; Taskaev, S.V.; Miki, H.; Takagi, T.; Vasiliev, A.N. Adiabatic temperature change at first-order magnetic phase transitions: $\mathrm{Ni}_{2.19} \mathrm{Mn}_{0.81} \mathrm{Ga}$ as a case study. Phys. Rev. B 2008, 78, doi:10.1103/PhysRevB.78.060403.

19. Fabbrici, S.; Porcari, G.; Cugini, F.; Solzi, M.; Kamarad, J.; Arnold, Z.; Cabassi, R.; Albertini, F. Co and In doped Ni-Mn-Ga magnetic shape memory alloys: A thorough structural, magnetic and magnetocaloric study. Entropy 2014, 16, 2204-2222.

20. Han, Z.D.; Qian, B.; Wang, D.H.; Zhang, P.; Jiang, X.F.; Zhang, C.L.; Du, Y.W. Magnetic phase separation and exchange bias in off-stoichiometric Ni-Mn-Ga alloys. Appl. Phys. Lett. 2013, 103, doi:10.1063/1.4826654.

21. Çakr, A.; Righi, L.; Albertini, F.; Acet, M.; Farle, M.; Aktúrk S. Extended investigation of intermartensitic transitions in Ni-Mn-Ga magnetic shape memory alloys: A detailed phase diagram determination. J. Appl. Phys. 2013, 114, doi:10.1063/1.4831667.

22. Wang, Y.; Huang, C.; Gao, J.; Yang, S.; Ding, X.; Song, X.; Ren, X. Evidence for ferromagnetic strain glass in Ni-Co-Mn-Ga Heusler alloy system. Appl. Phys. Lett. 2012, 101, doi:10.1063/1.4751250.

23. Wang, Y.; Huang, C.; Wu, H.; Gao, J.; Yang, S.; Wang, D.; Ding, X.; Song, X.; Ren, X. Spontaneous strain glass to martensite transition in ferromagnetic Ni-Co-Mn-Ga strain glass. Appl. Phys. Lett. 2013, 102, doi:10.1063/1.4799151.

24. Bozhko, A.D.; Vasiliev, A.N.; Khovailo, V.V.; Buchelnikov, V.D.; Dikshtein, I.E.; Seletskii, S.M.; Shavrov, V.G. Phase transitions in the ferromagnetic alloys $\mathrm{Ni}_{2+x} \mathrm{Mn}_{1-x} \mathrm{Ga}$. JETP Lett. 1998, 67, 227-232.

25. Jin, X.; Marioni, M.; Bono, D.; Allen, S.M.; O’Handley, R.C.; Hsu, T.Y. Empirical mapping of Ni-Mn-Ga properties with composition and valence electron concentration. J. Appl. Phys. 2002, 91, 8222-8224. 
26. Khovailo, V.V.; Novosad, V.; Takagi, T.; Filippov, D.A.; Levetin, R.Z.; Vasil'ev, A.N. Magnetic properties and magnetostructural phase transitions in $\mathrm{Ni}_{2+x} \mathrm{Mn}_{1-x} \mathrm{Ga}$ shape memory alloys. Phys. Rev. B 2004, 70, doi:10.1103/PhysRevB.70.174413.

27. Khovaylo, V.V.; Buchelnikov, V.D.; Kainuma, R.; Koledov, V.V.; Otsuka, M.; Shavrov, V.G.; Takagi, T.; Taskaev, S.V.; Vasiliev, A.N. Phase transitions in $\mathrm{Ni}_{2+x} \mathrm{Mn}_{1-x} \mathrm{Ga}$ with a high Ni excess. Phys. Rev. B 2005, 72, doi:10.1103/PhysRevB.72.224408.

28. Richard, M.; Feuchtwanger, J.; Schlagel, D.; Lograsso, T.; Allen, S.M; O’Handley, R.C. Crystal structure and transformation behavior of Ni-Mn-Ga martensites. Scr. Mater. 2006, 54, 1797-1801.

29. Li, Z.; Zhang, Y.; Esling, C.; Zhao, X.; Zuo, L. Twin relationships of 5M modulated martensite in Ni-Mn-Ga alloy. Acta Mater. 2011, 59, 3390-3397.

30. Ranjan, R.; Banik, S.; Barman, S.R.; Kumar, U.; Mukhopadhyay, P.K.; Pandey, D. Powder x-ray diffraction study of the thermoelastic martensitic transition on $\mathrm{Ni}_{2} \mathrm{Mn}_{1.05} \mathrm{Ga}_{0.95}$. Phys. Rev. B 2006, 74, doi:10.1103/PhysRevB.74.224443.

31. Righi, L.; Albertini, F.; Villa, E.; Paoluzi, A.; Calestani, G.; Chernenko, V.; Besseghini, S.; Ritter, C.; Passaretti, F. Crystal structure of 7M modulated Ni-Mn-Ga martensitic phase. Acta Mater. 2008, 56, 4529-4535.

32. Wedel, B.; Suzuki, M.; Murakami, Y.; Wedel, C.; Suzuki, T.; Shindo, D.; Itagaki, K. Low temperature crystal structure of Ni-Mn-Ga alloys. J. Alloys Compd. 1999, 290, 137-143.

33. Söderberg, O.; Brown, D.; Aaltio, I.; Oksanen, J.; Syrén, J.; Pulkkinen, H.; Hannula, S.P. Microstructure and properties of Ni-Mn-Ga alloys produced by rapid solidification and pulsed electric current sintering. J. Alloys Compd. 2011, 509, 5981-5987.

34. Khovailo, V.V.; Abe, T.; Koledov, V.V.; Matsumoto, M.; Nakamura, H.; Note, R.; Ohtsuka, M.; Shavrov, V.G.; Takagi, T. Influence of Fe and Co on Phase Transitions in Ni-Mn-Ga Alloys. Mater. Trans. 2003, 44, 2509-2512.

35. Cong, D.Y.; Wang, S.; Wang, Y.D.; Ren, Y.; Zuo, L.; Esling, C. Martensitic and magnetic transformation in Ni-Mn-Ga-Co ferromagnetic shape memory alloys. Mater. Sci. Eng. A 2008, 473, 213-218.

36. Ma, Y.; Yang, S.; Liu, Y.; Liu, X. The ductility and shape-memory properties of Ni-Mn-Co-Ga high-temperature shape-memory alloys. Acta Mater. 2009, 57, 3232-3241.

37. Glavatskyy, I.; Glavatska, N.; Söderberg, O.; Hannula, S.-P.; Hoffmann, J.-U. Transformation temperatures and magnetoplasticity of Ni-Mn-Ga alloyed with Si, In, Co or Fe. Scr. Mater. 2006, 54, 1891-1895.

38. Soto-Parra, D.E.; Moya, X.; Mañosa, L.; Planes, A.; Flores-Zúñiga, H.; Alvarado-Hernández, F.; Ochoa-Gamboa, R.A.; Matutes-Aquino, J.A.; Ríos-Jara, D. Fe and Co selective substitution in $\mathrm{Ni}_{2} \mathrm{MnGa}$ : Effect of magnetism on relative phase stability. Philos. Mag. 2010, 90, 2771-2792.

39. Rolfs, K.; Wimpory, R.C.; Petry, W.; Schneider, R. Effect of alloying Ni-Mn-Ga with Cobalt on thermal and structural properties. J. Phys. Conf. Ser. 2010, 251, doi:10.1088/1742-6596/ 251/1/012046.

40. Kumar, A.S.; Ramudu, M.; Seshubai, V. Effect of selective substitution of Co for Ni or Mn on the superstructure and microstructural properties of $\mathrm{Ni}_{50} \mathrm{Mn}_{29} \mathrm{Ga}_{21}$. J. Alloys Compd. 2011, 509, 8215-8222. 
41. Fabbrici, S.; Kamarad, J.; Arnold, Z.; Casoli, F.; Paoluzi, A.; Bolzoni, F.; Cabassi, R.; Solzi, M.; Porcari, G.; Pernechele, C.; et al. From direct to inverse giant magnetocaloric effect in Co-doped NiMnGa multifunctional alloys. Acta Mater. 2011, 59, 412-419.

42. Kumar, A.S.; Ramudu, M.; Seshubai, V. Structural and magnetic investigations in the vicinity of first-order transformations in Ni-Mn-Ga-Co ferromagnetic shape memory alloys. Phase Trans. 2012, 85, 1045-1059.

43. Segui, C.; Cesari, E. Composition and atomic order effects on the structural and magnetic transformations in ferromagnetic Ni-Co-Mn-Ga shape memory alloys. J. Appl. Phys. 2012, 111, doi:10.1063/1.3688019.

44. Pushin, V.G.; Korolev, A.V.; Kourov, N.I.; Kuranova, N.N.; Marchenkova, E.B.; Popov, A.G. On the effect of cobalt doping on thermoelastic martensitic transformations in ferromagnetic Heusler $\mathrm{Ni}_{50-x} \mathrm{Co}_{x} \mathrm{Mn}_{29} \mathrm{Ga}_{21}$ magnetically controlled shape memory alloys. Tech. Phys. Lett. 2013, 39, 737-740.

45. Khan, M.; Dubenko, I.; Stadler, S.; Ali, N. The structural and magnetic properties of $\mathrm{Ni}_{2} \mathrm{Mn}_{1-x} \mathrm{M}_{x} \mathrm{Ga}(\mathrm{M}=\mathrm{Co}, \mathrm{Cu})$. J. Appl. Phys. 2005, 97, doi:10.1063/1.1847131.

46. Kanomata, T.; Nunoki, S.; Endo, K.; Kataoka, M.; Nishihara, H.; Khovaylo, V.V.; Umetsu, R.Y.; Shishido, T.; Nagasako, M.; Kainuma, R.; et al. Phase diagram of the ferromagnetic shape memory alloys $\mathrm{Ni}_{2} \mathrm{MnGa}_{1 x} \mathrm{Co}_{x}$. Phys. Rev. B 2012, 85, doi:10.1103/PhysRevB.85.134421.

47. Kumar, A.S.; Ramudu, M.; Seshubai, V. Site preference of magnetic atoms in Ni-Mn-Ga-M ( $\mathrm{M}=\mathrm{Co}, \mathrm{Fe})$ ferromagnetic shape memory alloys. Phys. Status Solidi B 2012, 249. 620-626.

48. Porcari, G.; Fabbrici, S.; Pernechele, C.; Albertini, F.; Buzzi, M.; Paoluzi, A.; Kamarad, J.; Arnold, Z.; Solzi, M. Reverse magnetostructural transformation and adiabatic temperature change in Coand In-substituted Ni-Mn-Ga alloys. Phys. Rev. B 2012, 85, doi:10.1103/PhysRevB.85.024414.

49. Emre, B.; Yüce, S.; Stern-Taulats, E.; Planes, A.; Fabbrici, S.; Albertini, F.; Mañosa, L. Large reversible entropy change at the inverse magnetocaloric effect in Ni-Co-Mn-Ga-In magnetic shape memory alloys. J. Appl. Phys. 2013, 113, doi:10.1063/1.4808340.

50. Segui, C. Effects of the interplay between atomic and magnetic order on the properties of metamagnetic Ni-Co-Mn-Ga shape memory alloys. J. Appl. Phys. 2014, 115, doi:10.1063/1.4868055.

51. Entel, P.; Gruner, M.E.; Adeagbo, W.A.; Zayak, A.T. Magnetic field-induced changes in magnetic shape memory alloys. Mater. Sci. Eng. A 2008, 481-482, 258-261.

52. Tan, C.-L.; Jiang, J.-X.; Tian, X.-H.; Cai, W. Effect of Co on magnetic property and phase stability of Ni-Mn-Ga ferromagnetic shape-memory alloys: A first-principles study. Chin. Phys. B 2010, 19, doi:10.1088/1674-1056/19/10/107102.

53. Bai, J.; Raulot, J.-M.; Zhang, Y.; Esling, C.; Zhao, X.; Zuo, L. The effects of alloying element Co on Ni-Mn-Ga ferromagnetic shape memory alloys from first-principles calculations. Appl. Phys. Lett. 2011, 98, doi:10.1063/1.3582239.

54. Zelený, M.; Sozinov, A.; Straka, L.; Björkman, T.; Nieminen, R.M. First-principles study of Co- and $\mathrm{Cu}$-doped $\mathrm{Ni}_{2} \mathrm{MnGa}$ along the tetragonal deformation path. Phys. Rev. B 2014, 89, doi:10.1103/PhysRevB.89.184103. 
55. Buchelnikov, V.D.; Entel, P.; Taskaev, S.V.; Sokolovskiy, V.V.; Hucht, A.; Ogura, M.; Akai, H.; Gruner, M.E.; Nayak, S.K. Monte Carlo study of the influence of antiferromagnetic exchange interactions on the phase transitions of ferromagnetic Ni-Mn-X alloys ( $\mathrm{X}=\mathrm{In}, \mathrm{Sn}, \mathrm{Sb})$. Phys. Rev. B 2008, 78, doi:10.1103/PhysRevB.78.184427.

56. Entel, P.; Dannenberg, A.; Siewert, M.; Herper, H.C.; Gruner, M.E.; Buchelnikov, V.D.; Chernenko, V.V. Composition-dependent basics of smart Heusler materials from first-principles calculations. Mater. Sci. Forum 2011, 684, 1-29.

57. Li, C.-M.; Luo, H.-B.; Hu, Q.-M.; Yang, R.; Johansson, B.; Vitos, L. Role of magnetic and atomic ordering in the martensitic transformation of Ni-Mn-In from a first-principles study. Phys. Rev. B 2012, 86, doi:10.1103/PhysRevB.86.214205.

58. Sokolovskiy, V.V.; Buchelnikov, V.D.; Zagrebin, M.A.; Entel, P.; Sahool, S.; Ogura, M. First-principles investigation of chemical and structural disorder in magnetic $\mathrm{Ni}_{2} \mathrm{Mn}_{1+x} \mathrm{Sn}_{1-x}$ Heusler alloys. Phys. Rev. B 2012, 86, doi:10.1103/PhysRevB.86.134418.

59. Entel, P.; Siewert, M.; Gruner, M.E.; Herper, H.C.; Comtesse, D.; Arróyave, R.; Singh, N.; Talaparta, A.; Sokolovskiy, V.V.; Buchelnikov, V.D.; et al. Complex magnetic ordering as a driven mechanism of multifunctional properties of Heusler alloys from first-principles. Eur. Phys. J. B 2013, 86, 65-75.

60. Comtesse, D.; Gruner, M.E.; Ogura, M.; Sokolovskiy, V.V.; Buchelnikov, V.D.; Grünebohm, A.; Arróyave, R.; Singh, N.; Gottschall, T.; Gutfleisch, O.; et al. First-principles calculation of the instability leading to giant inverse magnetocaloric effects. Phys. Rev. B 2014, 89, doi:10.1103/PhysRevB.89.184403.

61. Castán, T.; Vives, E.; Lindgard, P.-A. Modeling premartensitic effects in $\mathrm{Ni}_{2} \mathrm{MnGa}$ : A mean-field and Monte Carlo simulation study. Phys. Rev. B 1999, 60, doi:10.1103/PhysRevB.60.7071.

62. Buchelnikov, V.D.; Sokolovskiy, V.V.; Herper, H.C.; Ebert, H.; Gruner, M.E.; Taskaev, S.V.; Khovaylo, V.V.; Hucht, A.; Dannenberg, A.; Ogura, M.; et al. First-principles and Monte Carlo study of magnetostructural transition and magnetocaloric properties of $\mathrm{Ni}_{2+x} \mathrm{Mn}_{1-x} \mathrm{Ga}$. Phys. Rev. B 2010, 81, doi:10.1103/PhysRevB.81.094411.

63. Buchelnikov, V.D.; Sokolovskiy, V.V.; Taskaev, S.V.; Khovaylo, V.V.; Aliev, A.A.; Khanov, L.N.; Batdalov, A.B.; Entel, P.; Miki, H.; Takagi, T. Monte Carlo simulations of the magnetocaloric effect in magnetic Ni-Mn-X (X = Ga, In) Heusler alloys. J. Phys. D Appl. Phys. 2011, 44, doi:10.1088/0022-3727/44/6/064012.

64. Sokolovskiy, V.V.; Buchelnikov, V.D.; Taskaev, S.V.; Khovaylo, V.V.; Ogura, M.; Entel, P. Quaternary Ni-Mn-In-Y Heusler alloys: A way to achieve materials with better magnetocaloric properties? J. Phys. D Appl. Phys. 2013, 46, doi:10.1088/0022-3727/46/30/305003.

65. Singh, N.; Arróyave, R. Magnetocaloric effects in Ni-Mn-Ga-Fe alloys using Monte Carlo simulations. J. Appl. Phys. 2013, 113, doi:10.1063/1.4803544.

66. Entel, P.; Gruner, M.; Comtesse, D.; Sokolovskiy, V.V; Buchelnikov, V.D. Interacting magnetic cluster-spin glasses and strain glasses in Ni-Mn based Heusler structured intermetallics. Phys. Status Solidi 2014, 1-14, doi:10.1002/pssb.201451059.

67. Ray, D.K.; Jardin, J.P. Elastic and magnetic interactions in a narrow twofold-degenerate band. Phys. Rev. B 1986, 33, 5021-5027. 
68. Murakami, Y.; Kainuma, R.; Shindo, D.; Tonomura, A. Magnetic microstructure analysis of ferromagnetic shape memory alloys and related compounds. Mater. Sci. Forum 2010, 684, $117-128$.

69. Ebert, H.; Ködderitzsch, D.; Minár, J. Calculating condensed matter properties using the KKR-Green's function method-recent developments and applications. Rep. Prog. Phys. 2011, 74, doi:10.1088/0034-4885/74/9/096501.

70. Ebert, H. SPR-KKR Package, version 6.3; allowing to calculate the electronic structure of various periodic systems, including in particular systems with chemical disorder; Available online: http://ebert.cup.unimuenchen.de (accessed on 17 September 2014).

71. Liechtenstein, A.I.; Katsnelson, M.I.; Antropov, V.P.; Gubanov, V.A. Local spin-density functional-approach to the theory of exchange interactions in ferromagnetic metals and alloys. $J$. Magn. Magn. Mater. 1987, 67, 65-74.

72. Perdew, J.P.; Burke, K.; Enzerhof, M. Generalized gradient approximation made simple. Phys. Rev. Lett. 1996, 77, 3865-3868.

73. Albertini, F.; Pareti, L.; Paoluzi, A.; Morellon, L.; Algaarabel, P.A.; Ibarra, M.R.; Righi, L. Composition and temperatue dependence of the magnetocrystalline anisotropy in $\mathrm{Ni}_{2+x} \mathrm{Mn}_{1-y} \mathrm{Ga}_{1-z}(x+y+z=0)$ Heusler alloys. Appl. Phys. Lett. 2002, 81, 4032-4034.

74. Enkovaara, J.; Ayuela, A.; Nordstöm, L.; Nieminen, R.M. Magnetic anisotropy in $\mathrm{Ni}_{2} \mathrm{MnGa}$. Phys. Rev. B 2002, 65, doi:10.1103/PhysRevB.65.134422.

75. Gruner, M.E.; Entel, P.; Opahle, I.; Richter, M. Ab initio investigation of twin boundary motion in the magnetic shape memory Heusler alloy $\mathrm{Ni}_{2} \mathrm{MnGa}$. J. Mater. Sci. 2008, 43, 3825-3831.

76. Şaşığlu, E.; Sandratskii, L.M.; Bruno, P. Role of conduction electrons in mediating exchange interactions in Mn-based Heusler alloys. Phys. Rev. B 2008, 77, doi:10.1103/PhysRevB.77.064417.

77. Anderson, P.W. Localized magnetic states in metals. Phys. Rev. B 1961, 124, doi:10.1103/ PhysRev.124.41.

78. Şaşığlu, E.; Sandratskii, L.M.; Bruno, P. First-principles calculation of the intersublattice exchange interactions and Curie temperatures of the full Heusler alloys $\mathrm{Ni}_{2} \mathrm{MnX}(\mathrm{X}=\mathrm{Ga}$, In, Sn, Sb). Phys. Rev. B 2004, 70, doi:10.1103/PhysRevB.70.024427.

79. Kurtulus, Y.; Dronskowski, R.; Samolyuk, G.D.; Antropov, V.P. Electronic structure and magnetic exchange coupling in ferromagnetic full Heusler alloys. Phys. Rev. B 2005, 71, doi:10.1103/PhysRevB.71.014425.

80. Rusz, J.; Bergqvist, L.; Kudrnovský, J.; Turek, I. Exchange interactions and Curie temperatures in $\mathrm{Ni}_{2-x} \mathrm{MnSb}$ alloys: First-principles study. Phys. Rev. B 2006, 73, doi:10.1103/PhysRevB. 73.214412.

81. Şaşığlu, E. Nonzero macroscopic magnetization in half-metallic antiferromagnets at finite temperatures. Phys. Rev. B 2009, 79, doi:10.1103/PhysRevB.79.100406.

82. Meinert, M.; Schmalhorst, J.-M.; Reiss, G. Ab initio prediction of ferrimagnetism, exchange interactions and Curie temperatures in $\mathrm{Mn}_{2}$ TiZ Heusler compounds. J. Phys. Condens. Matter 2011, 23, doi:10.1088/0953-8984/23/3/036001.

83. Meinert, M.; Schmalhorst, J.-M.; Reiss, G. Exchange interactions and Curie temperatures of $\mathrm{Mn}_{2} \mathrm{CoZ}$ compounds. J. Phys. Condens. Matter 2011, 23, doi:10.1088/0953-8984/23/11/116005. 
84. Da Silva, C.G.; Falicov, L.M. Theory of magnetic properties of rare earth compounds (Localized moments and hybridization effects). J. Phys. C 1972, 5, 63-76.

85. Shi, Z.-P.; Levy, P.M.; Fry, J.L. Interlayer magnetic coupling in metallic multilayer structures. Phys. Rev. B 1994, 49, doi:10.1103/PhysRevB.49.15159.

86. Ködderitzsch, D.; Ebert, H.; Rowlands, D.A.; Ernst, A. Relativistic formulation of the Korringa-Kohn-Rostoker nonlocal coherent-potential approximation. New J. Phys. 2007, 9, 81-17.

87. Özdoğan, K.; Şaşığlu, E.; Galanakis, I. Engineering the electronic, magnetic, and gap-related properties of the quinternary half-metallic Heusler alloys. J. Appl. Phys. 2008, 103, doi:10.1063/ 1.2831224.

88. Galanakis, I.; Şaşığlu, E. Variation of the magnetic properties of $\mathrm{Ni}_{2} \mathrm{MnGa}$ Heusler alloy upon tetragonalization: a first-principles study. J. Phys. D Appl. Phys. 2011, 44, doi:10.1088/0022-3727 /44/23/235001.

(c) 2014 by the authors; licensee MDPI, Basel, Switzerland. This article is an open access article distributed under the terms and conditions of the Creative Commons Attribution license (http://creativecommons.org/licenses/by/3.0/). 